\title{
Algebraic field theory operads and linear quantization
}

\author{
Simen Bruinsma ${ }^{1}\left[\mathbb{C}^{-}\right.$Alexander Schenkel ${ }^{1}$ \\ Received: 27 September 2018 / Revised: 30 April 2019 / Accepted: 3 July 2019 / Published online: 9 July 2019 \\ (c) The Author(s) 2019
}

\begin{abstract}
We generalize the operadic approach to algebraic quantum field theory (arXiv:1709.08657) to a broader class of field theories whose observables on a spacetime are algebras over any single-colored operad. A novel feature of our framework is that it gives rise to adjunctions between different types of field theories. As an interesting example, we study an adjunction whose left adjoint describes the quantization of linear field theories. We also analyze homotopical properties of the linear quantization adjunction for chain complex valued field theories, which leads to a homotopically meaningful quantization prescription for linear gauge theories.
\end{abstract}

Keywords Algebraic quantum field theory · Locally covariant quantum field theory · Colored operads · Universal constructions · Gauge theory · Model categories

Mathematics Subject Classification $81 \mathrm{Txx} \cdot 18 \mathrm{D} 50 \cdot 18 \mathrm{G} 55$

\section{Contents}

1 Introduction and summary . . . . . . . . . . . . . . . . . . . . . . . . . . 2532

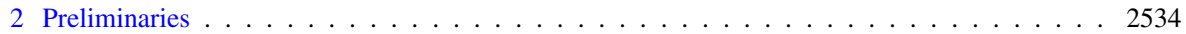

2.1 Colored operads . . . . . . . . . . . . . . . . . . . . . . . . . . . 2535

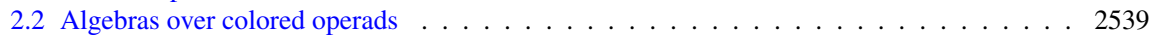

3 Field theory operads . . . . . . . . . . . . . . . . . . . . . . . . . . . . 2540

3.1 Orthogonal categories and field theories . . . . . . . . . . . . . . . . . . . 2540

3.2 Operadic description . . . . . . . . . . . . . . . . . . . . . . . . 2544

3.3 Functoriality . . . . . . . . . . . . . . . . . . . . . . . . . 2548

4 Universal constructions for field theories . . . . . . . . . . . . . . . . . . . . . . 2550

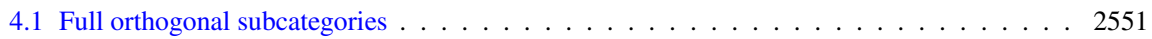

4.2 Orthogonal localizations . . . . . . . . . . . . . . . . . . . . 2553

Alexander Schenkel

alexander.schenkel@nottingham.ac.uk

Simen Bruinsma

simen.bruinsma@nottingham.ac.uk

1 School of Mathematical Sciences, University of Nottingham, University Park,

Nottingham NG7 2RD, UK 
4.3 Change of bipointed single-colored operad . . . . . . . . . . . . . . . . . . 2555

5 Linear quantization adjunction . . . . . . . . . . . . . . . . . . . . . . . . 2557

6 Toward the quantization of linear gauge theories . . . . . . . . . . . . . . . . . . . . 2560

6.1 Model structures on field theory categories . . . . . . . . . . . . . . . . . . . . 2561

6.2 Homotopical properties of linear quantization . . . . . . . . . . . . . . . . . . . 2562

6.3 Homotopy $j$-locality and homotopy $W$-constancy . . . . . . . . . . . . . . . . . . . . . 2564

A Technical details for Sect $6.2 \ldots \ldots \ldots \ldots$. . . . . . . . . . . . . . . . 2567

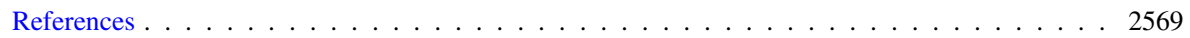

\section{Introduction and summary}

Algebraic quantum field theory is a well-established and successful framework to axiomatize and investigate quantum field theories on the Minkowski spacetime and also on more general Lorentzian manifolds, see e.g. [11,25] for overviews. In this setting a theory is described by a functor $\mathfrak{A}: \mathbf{C} \rightarrow \mathbf{A l g}_{\text {As }}$ from a suitable category $\mathbf{C}$ of spacetimes to the category of associative and unital algebras, which is required to satisfy some physically motivated axioms. For instance, in locally covariant algebraic quantum field theory $[13,18], \mathbf{C}=\mathbf{L o c}$ is the category of globally hyperbolic Lorentzian manifolds with morphisms given by causal isometric embeddings and the physical axioms are Einstein causality and the time-slice axiom. Einstein causality demands that any two observables, i.e. elements of the algebras assigned by $\mathfrak{A}$, that are causally disjoint commute with each other, which encodes the idea that two measurements in causally disjoint spacetime regions do not influence each other. The time-slice axiom demands that the algebra maps $\mathfrak{A}(f): \mathfrak{A}(M) \rightarrow \mathfrak{A}\left(M^{\prime}\right)$ associated with Cauchy morphisms, i.e. spacetime embeddings $f: M \rightarrow M^{\prime}$ such that $f(M) \subseteq M^{\prime}$ contains a Cauchy surface of $M^{\prime}$, are isomorphisms, which encodes a concept of time evolution. The framework of algebraic quantum field theory can also be adapted to obtain a novel point of view on classical field theories, see e.g. $[5,12,14,22]$, where in contrast to associative and unital algebras one assigns Poisson algebras of classical observables to spacetimes. The classical analog of Einstein causality then demands that the Poisson bracket between any two causally disjoint observables is zero.

The aim of this paper is to develop an operadic framework that generalizes [8] to a very broad and flexible class of field theories, see Definition 3.3. This includes as special instances the various flavors of algebraic quantum field theory $[11,13,18,25]$ and their classical analogs [5,12,14,22]. Our two main motivations for this work are as follows: (1) Describing field theories in terms of algebras over colored operads provides an excellent framework to discover and study universal constructions. This has already lead to a refinement of Fredenhagen's universal algebra construction for quantum field theories [19-21,31] in terms of a so-called operadic left Kan extension [8], which technically behaves better than the original construction as it respects the quantum field theory axioms. In this paper, we will show that the quantization of linear field theories may be expressed in terms of an operadic left Kan extension too, which allows us to understand and describe the interplay between quantization and other universal constructions. (2) Operadic techniques are particularly useful and powerful when working with chain complex valued field theories, e.g. gauge theories described 
in the BRST/BV formalism [22,23,29]. The reason for this is that chain complexes are naturally compared by quasi-isomorphisms and hence one is only allowed to perform constructions that preserve quasi-isomorphisms. Operad theory provides a huge toolbox to develop such constructions, which in technical language are called derived functors, see, e.g. [27,28] and also [9] for applications to quantum field theory. In this paper, we apply these techniques to investigate homotopical properties of the linear quantization functor. A similar construction in the context of factorization algebras [15] has been recently investigated in [24]. As a simple example, we present a quantization of linear Chern-Simons theory on oriented surfaces that is compatible with quasi-isomorphisms.

Let us now present a more detailed outline of the content of this paper. In Sect. 2, we shall fix our notations by recalling the necessary background material on colored operads and their algebras. In Sect. 3, we introduce our broad and flexible framework for field theories in Definition 3.3. A field theory is described by a functor $\mathfrak{A}: \mathbf{C} \rightarrow$ $\mathbf{A l g}_{\mathcal{P}}$ from a small category $\mathbf{C}$ to the category of algebras over a single-colored operad $\mathcal{P}$, which is required to satisfy a suitable generalization of the Einstein causality axiom. (The time-slice axiom will be formalized via localization techniques in Sect. 4.2.) One should interpret $\mathbf{C}$ as a category of spacetimes and $\mathcal{P}$ as the operad controlling the algebraic structure of the observables on a fixed spacetime. For example, quantum field theories are obtained by choosing the associative operad $\mathcal{P}=$ As and classical field theories by choosing the Poisson operad $\mathcal{P}=$ Pois. Linear field theories, which we describe in terms of Heisenberg Lie algebras of presymplectic vector spaces, are obtained by choosing the unital Lie operad $\mathcal{P}=$ uLie. One of the key results of this section (see Theorem 3.12) is that such field theories are precisely the algebras over a colored operad that we denote by $\mathcal{P}_{\overline{\mathbf{C}}}^{\left(r_{1}, r_{2}\right)}$. This colored operad depends on two different kinds of input data, which control the spacetime category of interest and the type of field theory. More precisely, the first datum is an orthogonal category $\overline{\mathbf{C}}=(\mathbf{C}, \perp)$ (see Definition 3.1), and the second is a bipointed single-colored operad $\mathcal{P}^{\left(r_{1}, r_{2}\right)}=\left(\mathcal{P}, r_{1}, r_{2}: I[2] \rightrightarrows \mathcal{P}\right)$ (see Definition 3.14). The orthogonality relation $\perp$ and the two pointings $r_{1}, r_{2}$ are required to formalize a suitable generalization of the Einstein causality axiom. We prove that the assignment $\left(\overline{\mathbf{C}}, \mathcal{P}^{\left(r_{1}, r_{2}\right)}\right) \mapsto \mathcal{P}_{\overline{\mathbf{C}}}^{\left(r_{1}, r_{2}\right)}$ of field theory operads is in a suitable sense functorial.

In Sect. 4, we harness this functorial behavior in order to study adjunctions between the categories of field theories corresponding to different $\overline{\mathbf{C}}$ and $\mathcal{P}^{\left(r_{1}, r_{2}\right)}$. This includes generalizations of the time-slicification and local-to-global adjunctions from [8], which have already found interesting applications to quantum field theory on spacetimes with boundaries [4]. A novel feature of our framework, which is not captured by [8], is a second kind of functorial assignment $\mathcal{P}^{\left(r_{1}, r_{2}\right)} \mapsto \mathcal{P}_{\overline{\mathbf{C}}}^{\left(r_{1}, r_{2}\right)}$ of our colored operads to bipointed single-colored operads. This results in adjunctions between the categories of field theories of different types. We shall investigate in detail the interplay of such adjunctions with the time-slice axiom and local-to-global property of field theories. A particularly interesting example, which we study in detail in Sect. 5, is given by an adjunction whose left adjoint describes the quantization of linear field theories. 
In Sect. 6, we extend our results to the case of $\mathbf{C h}(\mathbb{K})$-valued field theories, i.e. gauge theories, by using techniques from model category theory [17,30]. Our reformulation in Sect. 5 of the usual quantization of linear field theories in terms of (the left adjoint of) an adjunction is very valuable for studying the quantization of linear gauge theories. In particular, it allows us to construct a derived linear quantization functor which provides a homotopically meaningful quantization prescription for linear gauge theories in the sense that it maps weakly equivalent linear gauge theories to weakly equivalent quantum gauge theories. A deeper homotopical study of the building blocks of the derived linear quantization functor (see Appendix A) reveals that it can be modeled (up to a natural weak equivalence) by the underived linear quantization functor. From a computational point of view, this is a very pleasing result because it allows us to write down explicit formulas for the quantization of linear gauge theories. This will be illustrated by studying linear Chern-Simons theory on oriented surfaces. We conclude by analyzing in some detail the interplay between our (derived) linear quantization functor and suitable homotopical generalizations of the time-slice axiom and localto-global property of gauge theories.

\section{Preliminaries}

Throughout this paper we fix a closed symmetric monoidal category $\mathbf{M}$, which we further assume to be complete and cocomplete, i.e. all small limits and colimits exist in $\mathbf{M}$. The monoidal product is denoted by $\otimes: \mathbf{M} \times \mathbf{M} \rightarrow \mathbf{M}$, the monoidal unit by $I \in \mathbf{M}$ and the internal hom by $[-,-]: \mathbf{M}^{\mathrm{op}} \times \mathbf{M} \rightarrow \mathbf{M}$, where $(-)^{\text {op }}$ denotes the opposite category. The symmetric braiding is denoted by $\tau: m \otimes m^{\prime} \rightarrow m^{\prime} \otimes m$, for all $m, m^{\prime} \in \mathbf{M}$. We shall always suppress the associator and unitors and in particular simply write $m_{1} \otimes \cdots \otimes m_{n}$ for multiple tensor products of objects $m_{1}, \ldots, m_{n} \in \mathbf{M}$. Because $\mathbf{M}$ is by assumption cocomplete, there exists a Set-tensoring $\otimes:$ Set $\times \mathbf{M} \rightarrow$ $\mathbf{M}$, which we denote with abuse of notation by the same symbol as the monoidal product. Explicitly, for any set $S \in \mathbf{S e t}$ and $m \in \mathbf{M}$, we define

$$
S \otimes m:=\coprod_{s \in S} m \in \mathbf{M}
$$

where $\coprod$ is the coproduct in $\mathbf{M}$.

Example 2.1 A simple example of a bicomplete closed symmetric monoidal category is the Cartesian closed category Set of sets. Here $\otimes=\times$ is the Cartesian product, $I=\{*\}$ is any singleton set and $[S, T]=\operatorname{Map}(S, T)$ is the set of maps from $S$ to $T$. The symmetric braiding is given by the flip map $\tau: S \times T \rightarrow T \times S,(s, t) \mapsto(t, s)$.

Example 2.2 Another standard example of a bicomplete closed symmetric monoidal category is the category $\mathbf{V e c}_{\mathbb{K}}$ of vector spaces over a field $\mathbb{K}$. Here $\otimes$ is the usual tensor product of vector spaces, $I=\mathbb{K}$ is the 1 -dimensional vector space and $[V, W]=$ $\operatorname{Hom}_{\mathbb{K}}(V, W)$ is the vector space of linear maps from $V$ to $W$. The symmetric braiding is given by the flip map $\tau: V \otimes W \rightarrow W \otimes V, v \otimes w \mapsto w \otimes v$. 


\subsection{Colored operads}

We provide a brief review of those aspects of the theory of colored operads that are relevant for this work. We refer to $[8,10,34]$ for a more detailed presentation.

Let $\mathfrak{C} \in$ Set be a non-empty set, which we shall call the 'set of colors'. We will use the notation $\underline{c}:=\left(c_{1}, \ldots, c_{n}\right) \in \mathfrak{C}^{n}$ for elements of the $n$-fold product set.

Definition 2.3 A $\mathfrak{C}$-colored operad $\mathcal{O}$ with values in $\mathbf{M}$ is given by the following data:

- for each $n \geq 0$ and $(\underline{c}, t) \in \mathfrak{C}^{n+1}$, an object $\mathcal{O}\left(\begin{array}{l}t \\ c\end{array}\right) \in \mathbf{M}$ (called the object of operations from $\underline{c}$ to $t$ );

- for each $n \geq 0,(\underline{c}, t) \in \mathfrak{C}^{n+1}$ and permutation $\sigma \in \Sigma_{n}$, an M-morphism $\mathcal{O}(\sigma)$ : $\mathcal{O}\left(\begin{array}{l}t \\ c\end{array}\right) \rightarrow \mathcal{O}\left(\begin{array}{c}\bar{t} \\ \underline{c} \sigma\end{array}\right)$ (called the permutation action), where $\underline{c} \sigma:=\left(c_{\sigma(1)}, \ldots, c_{\sigma(n)}\right)$;

- for each $n>0, k_{1}, \ldots, k_{n} \geq 0,(\underline{a}, t) \in \mathfrak{C}^{n+1}$ and $\left(\underline{b}_{i}, a_{i}\right) \in \mathfrak{C}^{k_{i}+1}$, for $i=$ $1, \ldots, n$, an M-morphism $\gamma: \mathcal{O}\left(\begin{array}{l}t \\ a\end{array}\right) \otimes \bigotimes_{i=1}^{n} \mathcal{O}\left(\begin{array}{l}a_{i} \\ b_{i}\end{array}\right) \rightarrow \mathcal{O}\left(\begin{array}{l}t \\ b\end{array}\right)$ (called the operadic composition), where $\underline{b}:=\left(\underline{b}_{1}, \ldots, \underline{b}_{n}\right)$ is defined by concatenation;

- for each $c \in \mathfrak{C}$, an M-morphism $\mathbb{1}: I \rightarrow \mathcal{O}\left(\begin{array}{l}c \\ c\end{array}\right)$ (called the operadic unit).

These data are required to satisfy the standard permutation action, equivariance, associativity, and unitality axioms, see e.g. [34, Definition 11.2.1]. A morphism $\phi: \mathcal{O} \rightarrow \mathcal{P}$ between two $\mathfrak{C}$-colored operads $\mathcal{O}$ and $\mathcal{P}$ with values in $\mathbf{M}$ is a family of $\mathbf{M}$-morphisms

$$
\phi: \mathcal{O}\left(\begin{array}{l}
t \\
c
\end{array}\right) \longrightarrow \mathcal{P}\left(\begin{array}{l}
t \\
\underline{c}
\end{array}\right)
$$

for all $n \geq 0$ and $(\underline{c}, t) \in \mathfrak{C}^{n+1}$, that is compatible with the permutation actions $\phi \mathcal{O}(\sigma)=\mathcal{P}(\sigma) \phi$, the operadic compositions $\phi \gamma^{\mathcal{O}}=\gamma^{\mathcal{P}}\left(\phi \otimes \bigotimes_{i=1}^{n} \phi\right)$ and the operadic units $\phi \mathbb{1}^{\mathcal{O}}=\mathbb{1}^{\mathcal{P}}$. We denote the category of $\mathfrak{C}$-colored operads with values in $\mathbf{M}$ by $\mathbf{O} \mathbf{p}_{\mathfrak{C}}(\mathbf{M})$.

Colored operads generalize the concept of (enriched) categories in the following sense. In contrast to allowing only for 1-to-1 operations, such as the morphisms $\mathbf{C}\left(c, c^{\prime}\right)$ in a category $\mathbf{C}$, colored operads also describe $n$-to- 1 operations in terms of the objects of operations $\mathcal{O}\left(\begin{array}{l}t \\ c\end{array}\right)$. The operadic composition generalizes the usual categorical composition to operations of higher arity and the operadic unit is analogous to the identity morphisms in a category. Permutation actions are a new feature for operations of arity $\geq 2$ and they have no analog in ordinary category theory. The following example clarifies how every category defines a colored operad with only 1-ary operations.

Example 2.4 Let $\mathbf{C}$ be a small category and denote its set of objects by $\mathbf{C}_{0}$. The following construction defines a $\mathbf{C}_{0}$-colored operad $\operatorname{Diag}_{\mathbf{C}} \in \mathbf{O} \mathbf{p}_{\mathbf{C}_{0}}($ Set $)$ with values in $\mathbf{M}=$ Set, which is called the diagram operad of $\mathbf{C}$, see e.g. [10]. For $(\underline{c}, t) \in \mathbf{C}_{0}^{n+1}$, one defines the set of operations by

$$
\operatorname{Diag}_{\mathbf{C}}\left(\begin{array}{l}
t \\
c
\end{array}\right):= \begin{cases}\emptyset, & \text { for } n \neq 1, \\
\mathbf{C}(c, t), & \text { for } \underline{c}=c .\end{cases}
$$

The permutation action is uniquely fixed because $\Sigma_{1}=\{e\}$ is the trivial group. The only non-trivial operadic compositions are $\gamma: \operatorname{Diag}_{\mathbf{C}}\left(\begin{array}{l}t \\ a\end{array}\right) \times \operatorname{Diag}_{\mathbf{C}}\left(\begin{array}{l}a \\ b\end{array}\right) \rightarrow \operatorname{Diag}_{\mathbf{C}}\left(\begin{array}{l}t \\ b\end{array}\right)$ and 
they are given by composition of morphisms in the category $\mathbf{C}$. Finally, the operadic unit $\mathbb{1}:\{*\} \rightarrow \operatorname{Diag}_{\mathbf{C}}\left(\begin{array}{l}c \\ c\end{array}\right)$ is given by the identity morphisms in the category $\mathbf{C}$. One confirms that this defines a colored operad in the sense of Definition 2.3.

Many interesting examples of (colored) operads can be conveniently defined in terms of generators and relations, see e.g. the examples below. Let us briefly explain how this construction works. We denote by $\operatorname{Seq}_{\mathfrak{C}}(\mathbf{M})$ the category of $\mathfrak{C}$-colored (nonsymmetric) sequences with values in $\mathbf{M}$. An object $X \in \mathbf{S e q}_{\mathfrak{C}}(\mathbf{M})$ is a family of objects $X\left(\begin{array}{l}t \\ c\end{array}\right) \in \mathbf{M}$, for all $n \geq 0$ and $(\underline{c}, t) \in \mathfrak{C}^{n+1}$, and a $\operatorname{Seq}_{\mathfrak{C}}(\mathbf{M})$-morphism $f: X \rightarrow Y$ is a family of M-morphisms $f: X\left(\begin{array}{l}t \\ \underline{c}\end{array}\right) \rightarrow Y\left(\begin{array}{l}t \\ \underline{c}\end{array}\right)$, for all $n \geq 0$ and $(\underline{c}, t) \in \mathfrak{C}^{n+1}$. There exists a forgetful functor $U: \mathbf{O p}_{\mathfrak{C}}(\mathbf{M}) \rightarrow \mathbf{S e q}_{\mathfrak{C}}(\mathbf{M})$ that forgets the permutation action, operadic composition and operadic unit of a $\mathfrak{C}$-colored operad. This functor has a left adjoint which is called the free $\mathfrak{C}$-colored operad functor, i.e. we have an adjunction

$$
F: \operatorname{Seq}_{\mathfrak{C}}(\mathbf{M}) \rightleftarrows \mathbf{O p}_{\mathfrak{C}}(\mathbf{M}): U
$$

Given any choice of generators $G \in \mathbf{S e q}_{\mathfrak{C}}(\mathbf{M})$, we consider the corresponding free $\mathfrak{C}$-colored operad $F(G) \in \mathbf{O p}_{\mathfrak{C}}(\mathbf{M})$. In order to implement relations, we consider $R \in \mathbf{S e q}_{\mathfrak{C}}(\mathbf{M})$ together with two parallel $\mathbf{S e q}_{\mathfrak{C}}(\mathbf{M})$-morphisms $r_{1}, r_{2}: R \rightrightarrows U F(G)$. Note that because (2.4) is an adjunction, the latter is equivalent to two parallel $\mathbf{O} \mathbf{p}_{\mathfrak{C}}(\mathbf{M})$-morphisms $r_{1}, r_{2}: F(R) \rightrightarrows F(G)$, which we denote with abuse of notation by the same symbols. Because the category $\mathbf{O} \mathbf{p}_{\mathfrak{C}}(\mathbf{M})$ is cocomplete, the following construction defines a $\mathfrak{C}$-colored operad.

Definition 2.5 The $\mathfrak{C}$-colored operad presented by the generators $G \in \operatorname{Seq}_{\mathfrak{C}}(\mathbf{M})$ and relations $r_{1}, r_{2}: R \rightrightarrows U F(G)$ is defined as the coequalizer

$$
F(R) \stackrel{r_{1}}{\underset{r_{2}}{\longrightarrow}} F(G)-\rightarrow F(G) /\left(r_{1}=r_{2}\right)
$$

in $\mathbf{O} \mathbf{p}_{\mathfrak{C}}(\mathbf{M})$.

Example 2.6 Consider for the moment $\mathbf{M}=$ Set. The associative operad As $\epsilon$ $\mathbf{O} \mathbf{p}_{\{*\}}($ Set) is the single-colored operad (i.e. $\mathfrak{C}=\{*\}$ is a singleton) presented by the following generators and relations: We define the set of generators of arity $n$ by

$$
G(n):= \begin{cases}\{\eta\}, & \text { for } n=0 \\ \{\mu\}, & \text { for } n=2 \\ \emptyset, & \text { else }\end{cases}
$$

for all $n \geq 0$. The generator $\mu$ in arity 2 is interpreted as a multiplication operation and the generator $\eta$ in arity 0 as a unit element. To implement associativity and left/right unitality of these operations, we consider 


$$
R(n):= \begin{cases}\{\lambda, \rho\}, & \text { for } n=1 \\ \{a\}, & \text { for } n=3 \\ \emptyset, & \text { else }\end{cases}
$$

for all $n \geq 0$, together with the two $\operatorname{Seq}_{\{*\}}(\operatorname{Set})$-morphisms $r_{1}, r_{2}: R \rightarrow U F(G)$ defined by

$$
r_{1}:\left\{\begin{array}{l}
\lambda \longmapsto \gamma(\mu \otimes(\eta \otimes \mathbb{1})), \\
\rho \longmapsto \gamma(\mu \otimes(\mathbb{1} \otimes \eta)), \\
a \longmapsto \gamma(\mu \otimes(\mu \otimes \mathbb{1})),
\end{array} \quad, \quad r_{2}:\left\{\begin{array}{l}
\lambda \longmapsto \mathbb{1}, \\
\rho \longmapsto \mathbb{1}, \\
a \longmapsto \gamma(\mu \otimes(\mathbb{1} \otimes \mu)),
\end{array}\right.\right.
$$

where the operadic composition and unit are those of the free operad $F(G)$. The associative operad As $:=F(G) /\left(r_{1}=r_{2}\right) \in \mathbf{O} \mathbf{p}_{\{*\}}($ Set $)$ is defined as the corresponding coequalizer.

It is instructive and useful to visualize the generators and relations in terms of rooted trees. The generators are depicted by

$$
\mu=\chi, \quad \eta=\downarrow_{0},
$$

and the relations (in the order they appear in (2.8)) then read as

$$
\lambda=\left|\mathbb{1}, \quad \lambda_{0}=\right| \mathbb{1}, \quad \lambda=\lambda \text {. }
$$

Let us note that the associative operad can be defined in any bicomplete closed symmetric monoidal category M. Using the Set-tensoring (2.1) and the unit object $I \in \mathbf{M}$, we define generators $G \otimes I \in \mathbf{S e q}_{\{*\}}(\mathbf{M})$ and relations $r_{1} \otimes I, r_{2} \otimes I$ : $R \otimes I \rightarrow U F(G) \otimes I \cong U F(G \otimes I)$ in the category of $\mathbf{M}$-valued sequences $\operatorname{Seq}_{\{*\}}(\mathbf{M})$. The corresponding coequalizer then defines the M-valued associative operad As := $F(G \otimes I) /\left(r_{1} \otimes I=r_{2} \otimes I\right) \in \mathbf{O} \mathbf{p}_{\{*\}}(\mathbf{M})$.

Example 2.7 Consider for the moment $\mathbf{M}=\mathbf{V e c}_{\mathbb{K}}$. The Lie operad Lie $\in \mathbf{O p}_{\{*\}}\left(\mathbf{V e c}_{\mathbb{K}}\right)$ is the single-colored operad presented by the following generators and relations: There is only one generator of arity 2, the Lie bracket, that we depict by

$$
[\cdot, \cdot]=
$$

The relations are given by antisymmetry and the Jacobi identity<smiles>CC(I)C#CC(C)I</smiles>

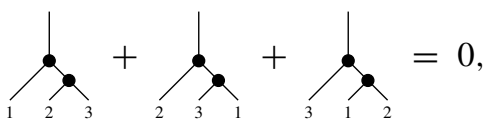


where the numbers below the trees indicate input permutations.

Note that for defining the Lie relations we had to use the natural Abelian group structure on the Hom-sets of $\mathbf{V e c}_{\mathbb{K}}$, i.e. addition of linear maps between vector spaces. Hence, the Lie operad can not be defined in a generic bicomplete closed symmetric monoidal category $\mathbf{M}$. If, however, $\mathbf{M}$ is an additive category, then one can define the Lie operad Lie $\in \mathbf{O} \mathbf{p}_{\{*\}}(\mathbf{M})$ with values in $\mathbf{M}$ along the same lines as above.

Example 2.8 As in Example 2.7, let us assume that $\mathbf{M}$ is additive. The Poisson operad Pois $\in \mathbf{O} \mathbf{p}_{\{*\}}(\mathbf{M})$ is the single-colored operad presented by the following generators and relations: The generators are

$$
\mu=\nearrow, \quad \eta=\downarrow_{0}, \quad\{\cdot, \cdot\}=\varnothing,
$$

where $\{\cdot, \cdot\}$ denotes the Poisson bracket. The generators $\mu$ and $\eta$ satisfy the relations of the associative operad (2.9) and the generator $\{\cdot, \cdot\}$ the relations of the Lie operad (2.10). We further demand the relations

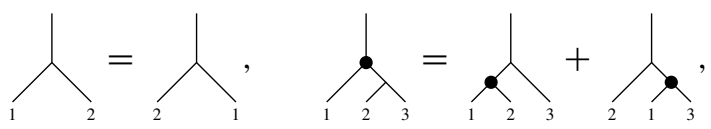

which express that $\mu$ is commutative and that $\{\cdot, \cdot\}$ is a derivation in the right entry (and hence by antisymmetry also a derivation in the left entry). Computing the operadic composition of the derivation relation with $\mathbb{1} \otimes \eta \otimes \eta$ implies that

$$
\ell_{0}=0
$$

i.e. the Poisson bracket of the unit element is zero.

Example 2.9 This example will play a role in the formalization of linear field theories; see Example 3.8. Let $\mathbf{M}$ be additive. The unital Lie operad uLie $\in \mathbf{O} \mathbf{p}_{\{*\}}(\mathbf{M})$ is the single-colored operad obtained by adding to the Lie operad from Example 2.7 a new generator of arity 0 , i.e. we have two generators

$$
[\cdot, \cdot]=\ell_{0}, \quad \eta=\downarrow
$$

In addition to the antisymmetry and Jacobi identity relations $(2.10)$ for $[\cdot, \cdot]$, we demand the compatibility relation

$$
\rho_{0}=0
$$

between the Lie bracket and the unit. 
We shall often require a generalization of the concept of colored operad morphisms from Definition 2.3 to morphisms that do not necessarily preserve the underlying sets of colors. As a preparation for the relevant definition, note that for every $\mathfrak{D}$-colored operad $\mathcal{P} \in \mathbf{O} \mathbf{p}_{\mathfrak{D}}(\mathbf{M})$ and every map of sets $f: \mathfrak{C} \rightarrow \mathfrak{D}$, one may define the pullback $\mathfrak{C}$-colored operad $f^{*}(\mathcal{P}) \in \mathbf{O} \mathbf{p}_{\mathfrak{C}}(\mathbf{M})$. Concretely, it is defined by setting $f^{*}(\mathcal{P})\left(\begin{array}{l}t \\ c\end{array}\right):=\mathcal{P}\left(\begin{array}{l}f(t) \\ f(\underline{c})\end{array}\right)$, for all $n \geq 0$ and $(\underline{c}, t) \in \mathfrak{C}^{n+1}$, and restricting the permutation action, operadic composition and operadic unit in the evident way.

Definition 2.10 The category $\mathbf{O p}(\mathbf{M})$ of operads with varying colors with values in $\mathbf{M}$ has as objects all pairs $(\mathfrak{C}, \mathcal{O})$ consisting of a non-empty set $\mathfrak{C}$ and a $\mathfrak{C}$-colored operad $\mathcal{O} \in \mathbf{O} \mathbf{p}_{\mathfrak{C}}(\mathbf{M})$. A morphism is a pair $(f, \phi):(\mathfrak{C}, \mathcal{O}) \rightarrow(\mathfrak{D}, \mathcal{P})$ consisting of a map of sets $f: \mathfrak{C} \rightarrow \mathfrak{D}$ and an $\mathbf{O p}_{\mathfrak{C}}(\mathbf{M})$-morphism $\phi: \mathcal{O} \rightarrow f^{*}(\mathcal{P})$ to the pullback $\mathfrak{C}$-colored operad.

\subsection{Algebras over colored operads}

We have seen above that a colored operad $\mathcal{O}$ describes abstract $n$-to- 1 operations, for all $n \geq 0$, together with a composition law $\gamma$, specified identities $\mathbb{1}$ and a permutation action $\mathcal{O}(\sigma)$ that allows us to permute the inputs of operations. Forming concrete realizations/representations of these abstract operations leads to the concept of algebras over colored operads.

Definition 2.11 An algebra A over a $\mathfrak{C}$-colored operad $\mathcal{O} \in \mathbf{O} \mathbf{p}_{\mathfrak{C}}(\mathbf{M})$, or shorter an $\mathcal{O}$-algebra, is given by the following data:

- for each $c \in \mathfrak{C}$, an object $A_{c} \in \mathbf{M}$;

- for each $n \geq 0$ and $(\underline{c}, t) \in \mathfrak{C}^{n+1}$, an M-morphism $\alpha: \mathcal{O}\left(\begin{array}{l}t \\ \underline{c}\end{array}\right) \otimes A_{\underline{c}} \rightarrow A_{t}$ (called $\mathcal{O}$-action), where $A_{\underline{c}}:=\bigotimes_{i=1}^{n} A_{c_{i}}$ with the convention that $A_{\emptyset}=I$ for $n=0$.

This data is required to satisfy the standard associativity, unity and equivariance axioms, see e.g. [34, Definition 13.2.3]. A morphism $\kappa: A \rightarrow B$ between two $\mathcal{O}$-algebras $A$ and $B$ is a family of M-morphisms $\kappa: A_{c} \rightarrow B_{c}$, for all $c \in \mathfrak{C}$, that is compatible with the $\mathcal{O}$-actions, i.e. $\kappa \alpha^{A}=\alpha^{B}\left(\mathrm{id} \otimes \bigotimes_{i=1}^{n} \kappa\right)$. We denote the category of $\mathcal{O}$-algebras by $\mathbf{A} \mathbf{l g}_{\mathcal{O}}$.

Example 2.12 Consider the diagram operad Diag $\mathbf{C} \in \mathbf{O p}_{\mathbf{C}_{0}}($ Set $)$ from Example 2.4.

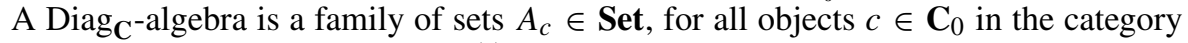
$\mathbf{C}$, together with maps $\alpha: \operatorname{Diag}_{\mathbf{C}}\left(\begin{array}{l}t \\ c\end{array}\right) \times A_{c} \rightarrow A_{t}$, for all $c, t \in \mathbf{C}_{0}$. (Here we already used that Diag ${ }_{\mathbf{C}}$ only contains 1-ary operations.) Because $\operatorname{Diag}_{\mathbf{C}}\left(\begin{array}{l}t \\ c\end{array}\right)=\mathbf{C}(c, t)$ is the Hom-set, the latter data is equivalent to specifying for each $\mathbf{C}$-morphism $f: c \rightarrow t$ a map of sets $A(f):=\alpha(f,-): A_{c} \rightarrow A_{t}$. The axioms for $\mathcal{O}$-algebras imply that $A(g f)=A(g) A(f)$, for all composable $\mathbf{C}$-morphism, and $A$ (id) $=$ id for the

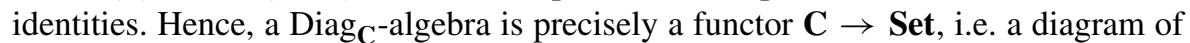
shape $\mathbf{C}$. One observes that morphisms between Diag $_{\mathbf{C}}$-algebras are precisely natural transformations between the corresponding functors.

Example 2.13 Consider for the moment $\mathbf{M}=$ Set and the associative operad As $\epsilon$ $\mathbf{O} \mathbf{p}_{\{*\}}($ Set $)$ from Example 2.6. An As-algebra is a single set $A=A_{*} \in$ Set 
together with an As-action. The latter is equivalent to providing a family of maps $\alpha: \operatorname{As}(n) \rightarrow \operatorname{Map}\left(A^{\times n}, A\right)$, for all $n \geq 0$, which define an $\mathbf{O p}_{\{*\}}(\mathbf{S e t})$-morphism to the endomorphism operad $\operatorname{End}(A)$, see e.g. [34, Definition 13.8.1]. Because As is presented by generators and relations (see Example 2.6), this is equivalent to defining $\alpha$ on the generators such that the relations hold true. This yields two maps $\mu_{A}:=\alpha(\mu): A \times A \rightarrow A$ and $\eta_{A}:=\alpha(\eta):\{*\} \rightarrow A$, which because of the relations have to satisfy the axioms of an associative and unital algebra in Set. One finds that morphisms of As-algebras are precisely morphisms of associative and unital algebras.

For a general bicomplete closed symmetric monoidal category $\mathbf{M}$, one obtains that the category $\mathbf{A l g}_{\text {As }}$ of algebras over As $\in \mathbf{O} \mathbf{p}_{\{*\}}(\mathbf{M})$ is the category of associative and unital algebras in $\mathbf{M}$. In particular, for $\mathbf{M}=\mathbf{V e c}_{\mathbb{K}}$, this is the category of associative and unital $\mathbb{K}$-algebras.

Example 2.14 A similar argument as in Example 2.13 shows that the category $\mathbf{A l g}_{\text {Lie }}$ of algebras over the Lie operad Lie $\in \mathbf{O} \mathbf{p}_{\{*\}}(\mathbf{M})$ (see Example 2.7) is the category of Lie algebras in $\mathbf{M}$ and that the category $\mathbf{A} \mathbf{I g}_{\text {Pois }}$ of algebras over the Poisson operad Pois $\in \mathbf{O} \mathbf{p}_{\{*\}}(\mathbf{M})$ (see Example 2.8) is the category of Poisson algebras in $\mathbf{M}$.

Given an $\mathbf{O p}(\mathbf{M})$-morphism $(f, \phi):(\mathfrak{C}, \mathcal{O}) \rightarrow(\mathfrak{D}, \mathcal{P})$ in the sense of Definition 2.10, one may define a pullback functor $(f, \phi)^{*}: \mathbf{A l g}_{\mathcal{P}} \rightarrow \mathbf{A l g}_{\mathcal{O}}$ between the corresponding categories of algebras. The pullback of $A \in \mathbf{A} \mathbf{l g}_{\mathcal{P}}$ is the $\mathcal{O}$-algebra defined by $\left((f, \phi)^{*} A\right)_{c}:=A_{f(c)} \in \mathbf{M}$, for all $c \in \mathfrak{C}$, together with the $\mathcal{O}$-action

$$
\mathcal{O}\left(\begin{array}{c}
t \\
\underline{c}
\end{array}\right) \otimes\left((f, \phi)^{*} A\right)_{\underline{c}} \stackrel{\phi \otimes \mathrm{id}}{\longrightarrow} \mathcal{P}\left(\begin{array}{c}
f(t) \\
f(\underline{c})
\end{array}\right) \otimes A_{f(\underline{c})} \stackrel{\alpha}{\longrightarrow} A_{f(t)}=\left((f, \phi)^{*} A\right)_{t} .
$$

Theorem 2.15 For any $\mathbf{O p}(\mathbf{M})$-morphism $(f, \phi):(\mathfrak{C}, \mathcal{O}) \rightarrow(\mathfrak{D}, \mathcal{P})$, the pullback functor $(f, \phi)^{*}: \mathbf{A l g}_{\mathcal{P}} \rightarrow \mathbf{A l g}_{\mathcal{O}}$ has a left adjoint, which is called operadic left Kan extension. We denote the corresponding adjunction by

$$
(f, \phi)_{!}: \mathbf{A l g}_{\mathcal{O}} \rightleftarrows \mathbf{A l g}_{\mathcal{P}}:(f, \phi)^{*}
$$

Example 2.16 Every functor $F: \mathbf{C} \rightarrow \mathbf{D}$ defines an evident $\mathbf{O p}(\mathbf{S e t})$-morphism $\left(F_{0}, F\right):\left(\mathbf{C}_{0}, \operatorname{Diag}_{\mathbf{C}}\right) \rightarrow\left(\mathbf{D}_{0}\right.$, Diag $\left._{\mathbf{D}}\right)$ between the corresponding diagram operads (see Example 2.4). Recalling from Example 2.12 that $\mathbf{A l g}_{\text {Diag }} \cong \mathbf{S e t}^{\mathbf{C}}$ is the category of functors from $\mathbf{C}$ to Set (and similarly that $\mathbf{A l g}_{\mathrm{Diag}_{\mathbf{D}}} \cong \mathbf{S e t}^{\mathbf{D}}$ ), one shows that the pullback functor $\left(F_{0}, F\right)^{*}$ is the usual pullback functor $F^{*}:=(-) \circ F: \mathbf{S e t}^{\mathbf{D}} \rightarrow \mathbf{S e t}^{\mathbf{C}}$ for functor categories. Its left adjoint $\left(F_{0}, F\right)$ ! is therefore the ordinary categorical left Kan extension $\operatorname{Lan}_{F}:$ Set $^{\mathbf{C}} \rightarrow$ Set $^{\mathbf{D}}$.

\section{Field theory operads}

\subsection{Orthogonal categories and field theories}

Let us briefly recall the basic idea of algebraic quantum field theory, see e.g. $[11,13,18$, 25] for more details. Broadly speaking, a field theory in this setting is a functor from 
a suitable category of spacetimes to a category of algebraic structures of interest that satisfies a list of physically motivated axioms. The prime example is given by functors $\mathfrak{A}:$ Loc $\rightarrow \mathbf{A l g}_{\text {As }}$ from the category Loc of globally hyperbolic Lorentzian manifolds to the category of associative and unital algebras that satisfy the Einstein causality axiom. The latter is a property of the functor $\mathfrak{A}: \mathbf{L o c} \rightarrow \mathbf{A l g}_{\text {As }}$ which demands that for every pair $\left(f_{1}: M_{1} \rightarrow M, f_{2}: M_{2} \rightarrow M\right)$ of Loc-morphisms whose images are causally disjoint in $M$ the diagram

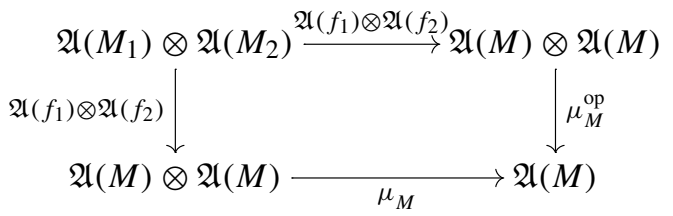

commutes, where $\mu_{M}^{(\mathrm{op})}$ denotes the (opposite) multiplication on $\mathfrak{A}(M)$. Another important physical example is given by functors $\mathfrak{A}: \mathbf{L o c} \rightarrow \mathbf{A l g}_{\text {As }}$ that satisfy the time-slice axiom in addition to the Einstein causality axiom. Such theories will be formalized later in Sect. 4.2 via localization techniques.

For the purpose of this paper, we consider the following generalization of quantum field theories satisfying the Einstein causality axiom. (Examples which justify this generalization are presented at the end of this subsection.) Let $\mathbf{C}$ be a small category which we interpret as a category of spacetimes. Instead of associative and unital algebras, let us take any single-colored operad $\mathcal{P} \in \mathbf{O} \mathbf{p}_{\{*\}}(\mathbf{M})$ and consider the functor category $\mathbf{A l g}_{\mathcal{P}} \mathbf{C}$. An object in this category is a functor $\mathfrak{A}: \mathbf{C} \rightarrow \mathbf{A l g}_{\mathcal{P}}$, i.e. an assignment of $\mathcal{P}$-algebras to spacetimes, and the morphisms are natural transformations between such functors. To encode physical axioms which generalize the Einstein causality axiom above, we recall the concept of orthogonal categories from [8].

Definition 3.1 An orthogonal category is a pair $\overline{\mathbf{C}}:=(\mathbf{C}, \perp)$ consisting of a small category $\mathbf{C}$ and a subset $\perp \subseteq$ Mor $\mathbf{C}_{\mathrm{t}} \times_{\mathrm{t}}$ Mor $\mathbf{C}$ of the set of pairs of morphisms with a common target, which satisfies the following properties:

(i) Symmetry: If $\left(f_{1}, f_{2}\right) \in \perp$, then $\left(f_{2}, f_{1}\right) \in \perp$.

(ii) Stability under compositions: If $\left(f_{1}, f_{2}\right) \in \perp$, then $\left(g f_{1} h_{1}, g f_{2} h_{2}\right) \in \perp$ for all composable C-morphisms $g, h_{1}$ and $h_{2}$.

We shall also write $f_{1} \perp f_{2}$ for elements $\left(f_{1}, f_{2}\right) \in \perp$. An orthogonal functor $F$ : $\overline{\mathbf{C}} \rightarrow \overline{\mathbf{D}}$ is a functor $F: \mathbf{C} \rightarrow \mathbf{D}$ that preserves orthogonality, i.e. if $f_{1} \perp_{\mathbf{C}} f_{2}$ then $F\left(f_{1}\right) \perp_{\mathbf{D}} F\left(f_{2}\right)$. We denote by OrthCat the category of orthogonal categories and orthogonal functors.

Example 3.2 Let Loc be any small category that is equivalent to the usual category of oriented, time-oriented and globally hyperbolic Lorentzian spacetimes of a fixed dimension $\geq 2$, see $[13,18]$. We define $\perp_{\text {Loc }}$ as the subset of all pairs $\left(f_{1}: M_{1} \rightarrow\right.$ $M, f_{2}: M_{2} \rightarrow M$ ) of causally disjoint Loc-morphisms, i.e. pairs of morphisms such that the images $f_{1}\left(M_{1}\right)$ and $f_{2}\left(M_{2}\right)$ are causally disjoint subsets in $M$. The pair $\overline{\mathbf{L o c}}:=\left(\mathbf{L o c}, \perp_{\mathbf{L o c}}\right)$ defines an orthogonal category. 
Let us now consider a parallel pair of $\mathbf{S e q}_{\{*\}}(\mathbf{M})$-morphisms $r_{1}, r_{2}: I[2] \rightrightarrows U(\mathcal{P})$, where $I[2] \in \operatorname{Seq}_{\{*\}}(\mathbf{M})$ is defined by

$$
I[2](n):= \begin{cases}I, & \text { for } n=2 \\ \emptyset, & \text { else }\end{cases}
$$

for all $n \geq 0$. This means that each $r_{i}$ picks out an operation of arity 2 in $\mathcal{P}$. For simplifying notation, we shall write

$$
\mathcal{P}^{\left(r_{1}, r_{2}\right)}:=\left(\mathcal{P}, r_{1}, r_{2}: I[2] \rightrightarrows U(\mathcal{P})\right)
$$

and we call $\mathcal{P}^{\left(r_{1}, r_{2}\right)}$ an (arity 2) bipointed single-colored operad.

Definition 3.3 A field theory of type $\mathcal{P}^{\left(r_{1}, r_{2}\right)}$ on $\overline{\mathbf{C}}$ is a functor $\mathfrak{A}: \mathbf{C} \rightarrow \mathbf{A l g}_{\mathcal{P}}$ that satisfies the following property: For all $\left(f_{1}: c_{1} \rightarrow c\right) \perp\left(f_{2}: c_{2} \rightarrow c\right)$, the diagram

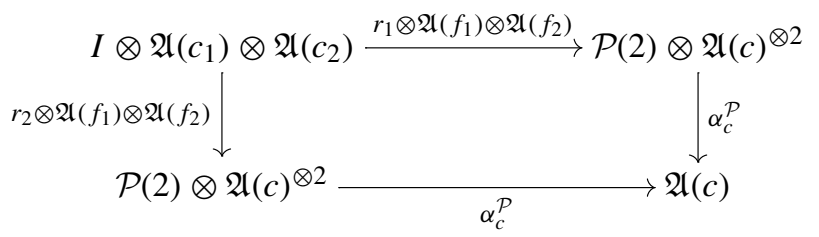

in $\mathbf{M}$ commutes, where $\alpha_{c}^{\mathcal{P}}$ denotes the $\mathcal{P}$-action on $\mathfrak{A}(c) \in \mathbf{A} \mathbf{I g}_{\mathcal{P}}$ (see Definition 2.11). The category of field theories of type $\mathcal{P}^{\left(r_{1}, r_{2}\right)}$ on $\overline{\mathbf{C}}$ is defined as the full subcategory

$$
\mathbf{F T}\left(\overline{\mathbf{C}}, \mathcal{P}^{\left(r_{1}, r_{2}\right)}\right) \subseteq \mathbf{A l g}_{\mathcal{P}} \mathbf{C}
$$

whose objects are all functors $\mathfrak{A}: \mathbf{C} \rightarrow \mathbf{A l g}_{\mathcal{P}}$ satisfying (3.4).

Remark 3.4 Our concept of field theories in Definition 3.3 is based on the idea that there exist two distinguished arity 2 operations in $\mathcal{P}$, which act in the same way when precomposed with an orthogonal pair $f_{1} \perp f_{2}$ of $\mathbf{C}$-morphisms. There exists an obvious generalization of this scenario to $n$-ary operations in $\mathcal{P}$ and orthogonal $n$-tuples of C-morphisms. We however decided not to introduce this more general framework for field theories, because all examples of interest to us are field theories in the sense of Definition 3.3.

Example 3.5 (Quantum field theories) Consider the associative operad As $\in \mathbf{O} \mathbf{p}_{\{*\}}(\mathbf{M})$

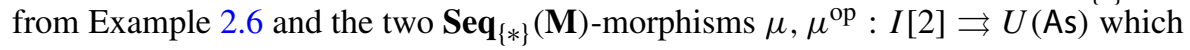
select the multiplication and opposite multiplication operations. A field theory of type $\mathrm{As}^{\left(\mu, \mu^{\mathrm{op}}\right)}$ on $\overline{\mathbf{C}}$ is a functor $\mathfrak{A}: \mathbf{C} \rightarrow \mathbf{A l g}_{\mathrm{As}}$ to the category of associative and unital algebras which satisfies the analog of (3.1). For $\overline{\mathbf{C}}=\overline{\text { Loc }}$ (see Example 3.2), this is a locally covariant quantum field theory $[13,18]$ that satisfies the Einstein causality axiom but not necessarily the time-slice axiom. The time-slice axiom will be discussed in Sect. 4.2. 
Remark 3.6 If $\mathbf{M}$ is additive, there exists an alternative but equivalent formalization of the type of field theories from Example 3.5. Consider the associative operad As $\in$ $\mathbf{O} \mathbf{p}_{\{*\}}(\mathbf{M})$ and the two $\mathbf{S e q}_{\{*\}}(\mathbf{M})$-morphisms [·, $\left.\cdot\right], 0: I[2] \rightrightarrows U($ As) which select the commutator $[\cdot, \cdot]=\mu-\mu^{\mathrm{op}}$ and the zero-operation (of arity 2 ). A field theory of type $A s^{([\cdot, \cdot], 0)}$ on $\overline{\mathbf{C}}$ is a functor $\mathfrak{A}: \mathbf{C} \rightarrow \mathbf{A l g}_{\mathrm{As}}$ to the category of associative and unital algebras which satisfies the property that

$$
\left[\mathfrak{A}\left(f_{1}\right)(-), \mathfrak{A}\left(f_{2}\right)(-)\right]_{c}: \mathfrak{A}\left(c_{1}\right) \otimes \mathfrak{A}\left(c_{2}\right) \longrightarrow \mathfrak{A}(c)
$$

is the zero-map, for all $\left(f_{1}: c_{1} \rightarrow c\right) \perp\left(f_{2}: c_{2} \rightarrow c\right)$. (Here $[\cdot, \cdot]_{c}=\mu_{c}-\mu_{c}^{\text {op }}$ denotes the commutator on $\mathfrak{A}(c)$.) This is equivalent to our description in Example 3.5 , i.e.

$$
\mathbf{F T}\left(\overline{\mathbf{C}}, \mathrm{As}^{([\cdot, \cdot], 0)}\right) \cong \mathbf{F T}\left(\overline{\mathbf{C}}, \mathrm{As}^{\left(\mu, \mu^{\mathrm{op}}\right)}\right) .
$$

This observation will be useful in Sect. 5 when we study the linear quantization adjunction.

Example 3.7 (Classical field theories) Let $\mathbf{M}$ be additive. Consider the Poisson operad Pois $\in \mathbf{O p}_{\{*\}}(\mathbf{M})$ from Example 2.8 and the two $\mathbf{S e q}_{\{*\}}(\mathbf{M})$-morphisms $\{\cdot, \cdot\}, 0$ : $I[2] \rightrightarrows U$ (Pois) which select the Poisson bracket and the zero-operation. A field theory of type Pois ${ }^{(\{\cdot, \cdot\}, 0)}$ on $\overline{\mathbf{C}}$ is a functor $\mathfrak{A}: \mathbf{C} \rightarrow \mathbf{A l g}_{\text {Pois }}$ to the category of Poisson algebras which satisfies the property that

$$
\left\{\mathfrak{A}\left(f_{1}\right)(-), \mathfrak{A}\left(f_{2}\right)(-)\right\}_{c}: \mathfrak{A}\left(c_{1}\right) \otimes \mathfrak{A}\left(c_{2}\right) \longrightarrow \mathfrak{A}(c)
$$

is the zero-map, for all $\left(f_{1}: c_{1} \rightarrow c\right) \perp\left(f_{2}: c_{2} \rightarrow c\right)$. (Here $\{\cdot, \cdot\}_{c}$ denotes the Poisson bracket on $\mathfrak{A}(c)$.) For $\overline{\mathbf{C}}=\overline{\mathbf{L o c}}$, this is a classical analog of locally covariant quantum field theory, where one assigns to each spacetime a Poisson algebra of classical observables, see e.g. [5,12,14,22]. The property (3.8) demands that the Poisson bracket between causally disjoint classical observables is zero, which captures the classical analog of the Einstein causality axiom.

Example 3.8 (Linear field theories) In the usual construction of linear quantum field theories, see e.g. [1-3] for reviews, one first defines a functor $\mathfrak{L}:$ Loc $\rightarrow$ PSymp to the category of presymplectic vector spaces, which is then quantized by forming CCR-algebras (CCR stands for canonical commutation relations). Recall that a presymplectic vector space $(V, \omega)$ is a pair consisting of a vector space $V$ and an antisymmetric linear map $\omega: V \otimes V \rightarrow \mathbb{K}$. Notice that this is not an operation of arity 2 in the sense of operads because the target is the ground field and not $V$. Hence, PSymp is not the category of algebras over an operad and, as a consequence, functors $\mathfrak{L}:$ Loc $\rightarrow$ PSymp do not define field theories in the sense of Definition 3.3.

However, there exists a canonical upgrade of every functor $\mathfrak{L}:$ Loc $\rightarrow$ PSymp to a field theory in the sense of Definition 3.3. Given any presymplectic vector space $(V, \omega)$, one can define its Heisenberg Lie algebra $H(V, \omega)$. The underlying vector space of 
$H(V, \omega)$ is given by $V \oplus \mathbb{K}$ and the Lie bracket $[-,-]:(V \oplus \mathbb{K}) \otimes(V \oplus \mathbb{K}) \rightarrow V \oplus \mathbb{K}$ is

$$
\left[v \oplus k, v^{\prime} \oplus k^{\prime}\right]:=0 \oplus \omega\left(v, v^{\prime}\right)
$$

for all $v \oplus k, v^{\prime} \oplus k^{\prime} \in V \oplus \mathbb{K}$. There exists a canonical unit map $\eta: \mathbb{K} \rightarrow V \oplus \mathbb{K}, k \mapsto$ $0 \oplus k$, which is compatible with the Lie bracket, i.e. $\left[v \oplus k, \eta\left(k^{\prime}\right)\right]=0$, for all $v \oplus k \in V \oplus \mathbb{K}$ and $k^{\prime} \in \mathbb{K}$. Hence, Heisenberg Lie algebras are algebras over the unital Lie operad uLie $\in \mathbf{O} \mathbf{p}_{\{*\}}(\mathbf{M})$ given in Example 2.9. Because forming Heisenberg Lie algebras is functorial, we can define for every $\mathfrak{L}:$ Loc $\rightarrow$ PSymp the composite functor $H \mathfrak{L}:$ Loc $\rightarrow \mathbf{A l g}_{\text {uLie }}$.

Consider now the two $\mathbf{S e q}_{\{*\}}(\mathbf{M})$-morphisms [·, $\left.\cdot\right], 0: I[2] \rightarrow U$ (uLie) which select the Lie bracket and the zero-operation. A field theory of type uLie ${ }^{([\cdot, \cdot], 0)}$ on $\overline{\mathbf{C}}$ is a functor $\mathfrak{A}: \mathbf{C} \rightarrow \mathbf{A l g}_{\text {uLie }}$ to the category of unital Lie algebras which satisfies the property that

$$
\left[\mathfrak{A}\left(f_{1}\right)(-), \mathfrak{A}\left(f_{2}\right)(-)\right]_{c}: \mathfrak{A}\left(c_{1}\right) \otimes \mathfrak{A}\left(c_{2}\right) \longrightarrow \mathfrak{A}(c)
$$

is the zero-map, for all $\left(f_{1}: c_{1} \rightarrow c\right) \perp\left(f_{2}: c_{2} \rightarrow c\right)$. (Here $[\cdot, \cdot]_{c}$ denotes the Lie bracket on $\mathfrak{A}(c)$.) This property is a suitable analog of the Einstein causality axiom for linear field theories. In particular, if $\overline{\mathbf{C}}=\overline{\mathbf{L o c}}, \mathbf{M}=\mathbf{V e c}_{\mathbb{K}}$ and $\mathfrak{A}=H \mathfrak{L}$ : $\mathbf{L o c} \rightarrow \mathbf{A l g}_{\text {uLie }}$ is given by applying the Heisenberg Lie algebra construction to a functor $\mathfrak{L}: \mathbf{L o c} \rightarrow$ PSymp with values in presymplectic vector spaces, then (3.10) is equivalent to the property that the presymplectic structure of causally disjoint linear observables is zero. This is precisely the Einstein causality axiom for linear field theories, see e.g. [1-3].

\subsection{Operadic description}

In this section, we show that the category of field theories from Definition 3.3 is the category of algebras over a suitable colored operad. This generalizes previous results in [8] and it is the key insight that allows us to study a large family of universal constructions for field theories in Sect. 4. As a preparation for the relevant definition, we define an auxiliary colored operad that describes functors from a small category $\mathbf{C}$ to the category of $\mathcal{P}$-algebras.

Definition 3.9 Let $\mathbf{C}$ be a small category with set of objects $\mathbf{C}_{0}$ and let $\mathcal{P} \in \mathbf{O} \mathbf{p}_{\{*\}}(\mathbf{M})$ be a single-colored operad. The $\mathbf{C}_{0}$-colored operad $\mathcal{P}_{\mathbf{C}} \in \mathbf{O p}_{\mathbf{C}_{0}}(\mathbf{M})$ is defined by the following data:

- for $n \geq 0$ and $(\underline{c}, t) \in \mathbf{C}_{0}^{n+1}$, the object of operations is

$$
\mathcal{P}_{\mathbf{C}}\left(\begin{array}{l}
t \\
c
\end{array}\right):=\mathbf{C}(\underline{c}, t) \otimes \mathcal{P}(n) \in \mathbf{M},
$$

where $\otimes$ is the Set-tensoring (2.1) and $\mathbf{C}(\underline{c}, t):=\prod_{i=1}^{n} \mathbf{C}\left(c_{i}, t\right)$ is the product of Hom-sets; 
- for $n \geq 0,(\underline{c}, t) \in \mathbf{C}_{0}^{n+1}$ and $\sigma \in \Sigma_{n}$, the permutation action $\mathcal{P}_{\mathbf{C}}(\sigma)$ is defined by

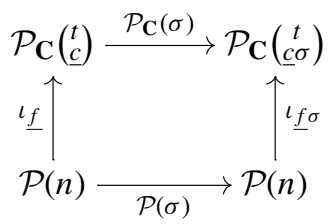

for all $f:=\left(f_{1}, \ldots, f_{n}\right) \in \mathbf{C}(\underline{c}, t)$, where $\iota_{f}: \mathcal{P}(n) \rightarrow \mathcal{P}_{\mathbf{C}}\left(\begin{array}{l}t \\ c\end{array}\right)=\mathbf{C}(\underline{c}, t) \otimes$ $\mathcal{P}(n)$ are the inclusion morphisms into the coproduct (see (2.1)) and $\underline{f} \sigma:=$ $\left(f_{\sigma(1)}, \ldots, f_{\sigma(n)}\right)$

- for $n>0, k_{1}, \ldots, k_{n} \geq 0,(\underline{a}, t) \in \mathbf{C}_{0}^{n+1}$ and $\left(\underline{b}_{i}, a_{i}\right) \in \mathbf{C}_{0}^{k_{i}+1}$, for $i=1, \ldots, n$, the operadic composition $\gamma^{\mathcal{P}_{\mathbf{C}}}$ is defined by

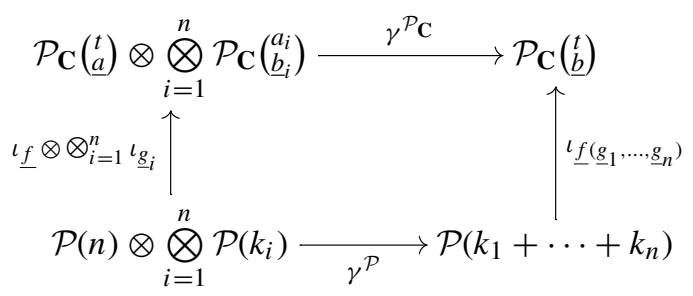

for all $\underline{f}=\left(f_{1}, \ldots, f_{n}\right) \in \mathbf{C}(\underline{a}, t)$ and $\underline{g}_{i}=\left(g_{i 1}, \ldots, g_{i k_{i}}\right) \in \mathbf{C}\left(\underline{b}_{i}, a_{i}\right)$, for $i=1, \ldots, n$, where $\underline{f}\left(\underline{g}_{1}, \ldots, \underline{g}_{n}\right):=\left(f_{1} \underline{g}_{11}, \ldots, f_{n} g_{n k_{n}}\right) \in \mathbf{C}(\underline{b}, t)$ is defined by composition in the category $\overline{\mathbf{C}}$;

- for $c \in \mathbf{C}_{0}$, the operadic unit $\mathbb{1}^{\mathcal{P}_{\mathbf{C}}}$ is

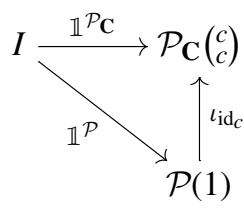

where $\operatorname{id}_{c}: c \rightarrow c$ is the identity morphism of $c$ in the category $\mathbf{C}$.

A straightforward check shows that these data define a colored operad (see Definition 2.3).

Lemma 3.10 There exists a canonical isomorphism

$$
\operatorname{Alg}_{\mathcal{P}_{\mathbf{C}}} \cong \operatorname{Alg}_{\mathcal{P}} \mathrm{C}
$$

between the category of algebras over the colored operad $\mathcal{P}_{\mathbf{C}} \in \mathbf{O}_{\mathbf{p}_{\mathbf{C}_{0}}}(\mathbf{M})$ from Definition 3.9 and the category of functors from $\mathbf{C}$ to $\mathbf{A l g}_{\mathcal{P}}$. 
Proof A $\mathcal{P}_{\mathbf{C}}$-algebra is a family of objects $A_{c} \in \mathbf{M}$, for all $c \in \mathbf{C}_{0}$, together with a

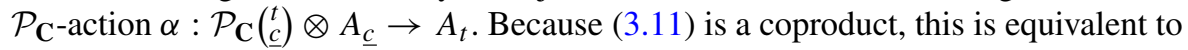
a family of M-morphisms $\alpha_{f}: \mathcal{P}(n) \otimes A_{\underline{c}} \rightarrow A_{t}$, for all $n \geq 0,(\underline{c}, t) \in \mathbf{C}_{0}^{n+1}$ and $f \in \mathbf{C}(\underline{c}, t)$, which satisfies the following compatibility conditions resulting from the axioms for algebras over colored operads
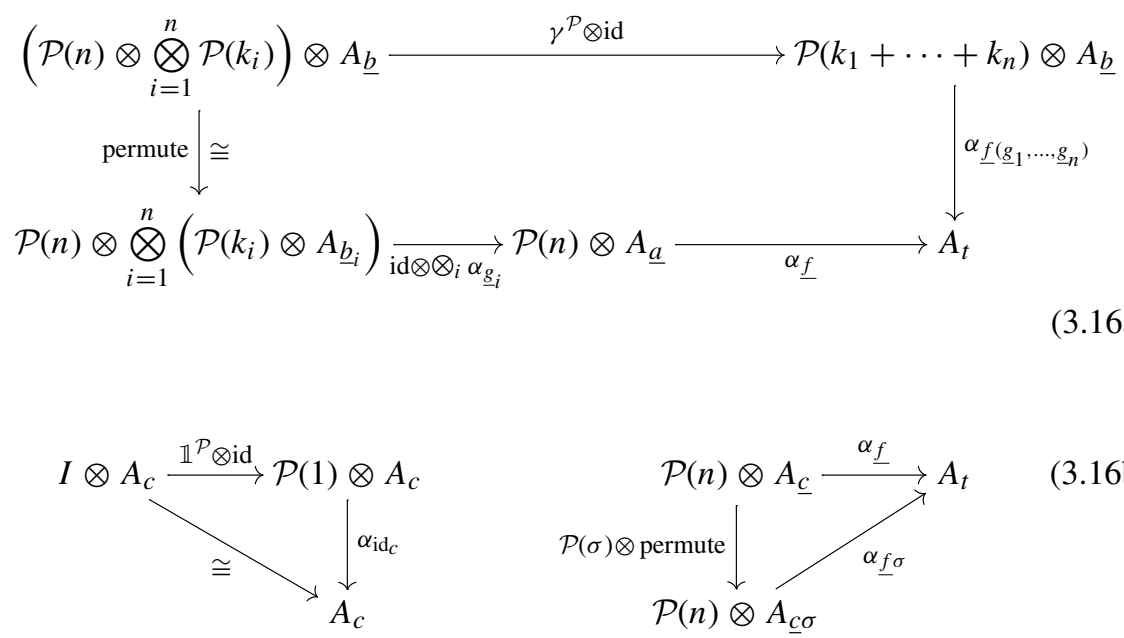

Using that any $f=\left(f_{1}, \ldots, f_{n}\right) \in \mathbf{C}(\underline{c}, t)$ can be written as $f=\operatorname{id}_{t}{ }^{n}\left(f_{1}, \ldots, f_{n}\right)$, where $\mathrm{id}_{t}^{n}=\left(\overline{\mathrm{id}}_{t}, \ldots, \mathrm{id}_{t}\right)$ is of length $\bar{n}$, the diagram (3.16a) $\overline{\mathrm{im}}$ plies that $\alpha_{\underline{f}}$ factorizes as

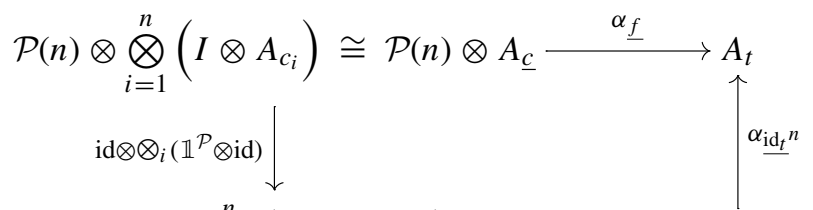

$$
\begin{aligned}
& \mathcal{P}(n) \otimes \bigotimes_{i=1}^{n}\left(\mathcal{P}(1) \otimes A_{c_{i}}\right) \underset{\operatorname{id} \otimes \otimes_{i} \alpha_{f_{i}}}{\longrightarrow} \mathcal{P}(n) \otimes A_{t}^{\otimes n}
\end{aligned}
$$

Hence, the $\mathcal{P}_{\mathbf{C}}$-action $\alpha$ is uniquely specified by the following two types of $\mathbf{M}$ morphisms: (1) $\tilde{\alpha}_{t}:=\alpha_{\mathrm{id}_{t}{ }^{n}}: \mathcal{P}(n) \otimes A_{t}^{\otimes n} \rightarrow A_{t}$, for all $t \in \mathbf{C}_{0}$ and $n \geq 0$, and (2) $A(f):=\alpha_{f}\left(\mathbb{1}^{\mathcal{P}} \otimes \mathrm{id}\right): A_{c} \cong I \otimes A_{c} \rightarrow A_{t}$, for all C-morphisms $f: c \rightarrow t$. The remaining conditions in (3.16) are equivalent to $\widetilde{\alpha}_{t}$ defining a $\mathcal{P}$-action on $A_{t}$, for all $t \in \mathbf{C}_{0}$, and $A(f): A_{c} \rightarrow A_{t}$ defining a functor $\mathbf{C} \rightarrow \mathbf{A l g} \mathbf{g}_{\mathcal{P}}$ to $\mathcal{P}$-algebras. From this perspective, $\mathcal{P}_{\mathbf{C}}$-algebra morphisms correspond precisely to natural transformations between functors from $\mathbf{C}$ to $\mathbf{A l g} \mathbf{P}_{\mathcal{P}}$.

For the rest of this subsection, we fix an orthogonal category $\overline{\mathbf{C}}=(\mathbf{C}, \perp)$ and a bipointed single-colored operad $\mathcal{P}^{\left(r_{1}, r_{2}\right)}=\left(\mathcal{P}, r_{1}, r_{2}: I[2] \rightrightarrows U(\mathcal{P})\right)$. (Recall the definition of $I$ [2] in (3.2).) We define a $\mathbf{C}_{0}$-colored sequence $R_{\perp} \in \mathbf{S e q}_{\mathbf{C}_{0}}(\mathbf{M})$ by setting 


$$
R_{\perp}\left(\begin{array}{l}
t \\
\underline{c}
\end{array}\right):= \begin{cases}(\perp \cap \mathbf{C}(\underline{c}, t)) \otimes I, & \text { for } n=2, \\
\emptyset, & \text { else },\end{cases}
$$

for all $n \geq 0$ and $(\underline{c}, t) \in \mathbf{C}_{0}^{n+1}$, and a parallel pair of $\mathbf{S e q}_{\mathbf{C}_{0}}(\mathbf{M})$-morphisms

$$
r_{1, \mathbf{C}}, r_{2, \mathbf{C}}: R_{\perp} \Longrightarrow U\left(\mathcal{P}_{\mathbf{C}}\right)
$$

by setting, for $i=1,2$,

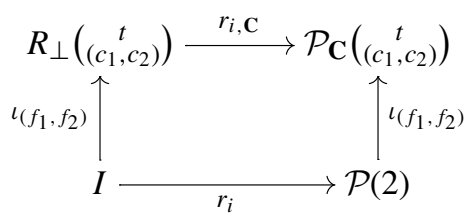

for all $\left(f_{1}: c_{1} \rightarrow t, f_{2}: c_{2} \rightarrow t\right) \in \perp$.

Definition 3.11 The operad of field theories of type $\mathcal{P}^{\left(r_{1}, r_{2}\right)}$ on $\overline{\mathbf{C}}$ is defined as the coequalizer

$$
F\left(R_{\perp}\right) \underset{r_{2, \mathbf{C}}}{\stackrel{r_{1, \mathbf{C}}}{\longrightarrow}} \mathcal{P} \mathbf{C}-\rightarrow \mathcal{P}_{\overline{\mathbf{C}}}^{\left(r_{1}, r_{2}\right)}
$$

in $\mathbf{O} \mathbf{p}_{\mathbf{C}_{0}}(\mathbf{M})$.

The importance of this operad is evidenced by the following theorem.

Theorem 3.12 There exists a canonical isomorphism

$$
\operatorname{Alg}_{\mathcal{P}_{\overline{\mathbf{C}}}^{\left(r_{1}, r_{2}\right)}} \cong \mathbf{F T}\left(\overline{\mathbf{C}}, \mathcal{P}^{\left(r_{1}, r_{2}\right)}\right)
$$

between the category of algebras over the colored operad $\mathcal{P}_{\overline{\mathbf{C}}}^{\left(r_{1}, r_{2}\right)} \in \mathbf{O}_{\mathbf{P}_{0}}(\mathbf{M})$ from Definition 3.11 and the category of field theories of type $\mathcal{P}^{\left(r_{1}, r_{2}\right)}$ on $\overline{\mathbf{C}}$ from Definition 3.3 .

Proof Because $\mathcal{P}_{\overline{\mathbf{C}}}^{\left(r_{1}, r_{2}\right)}$ is defined as a coequalizer (3.20), its algebras are precisely those $\mathcal{P}_{\mathbf{C}}$-algebras $A \in \mathbf{A} \mathbf{I g}_{\mathcal{P}_{\mathbf{C}}}$ that satisfy the relations encoded by $r_{1}, \mathbf{C}, r_{2}, \mathbf{C}: R_{\perp} \rightrightarrows$ $U\left(\mathcal{P}_{\mathbf{C}}\right)$ [see (3.19)]. Using the notations from the proof of Lemma 3.10, this concretely means that the diagram

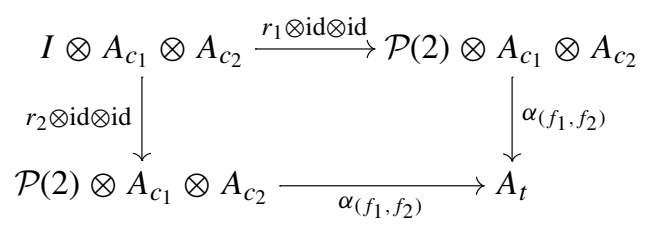


in $\mathbf{M}$ commutes, for all $\left(f_{1}: c_{1} \rightarrow t, f_{2}: c_{2} \rightarrow t\right) \in \perp$. Using the isomorphism of Lemma 3.10, one easily translates this diagram to the diagram (3.4) for the functor $\mathfrak{A}: \mathbf{C} \rightarrow \mathbf{A l g}_{\mathcal{P}}$ corresponding to $A \in \mathbf{A l g}_{\mathcal{P}_{\mathbf{C}}}$, which completes the proof.

Example 3.13 Recalling Examples 3.5, 3.7 and 3.8, our construction defines colored operads for quantum field theory $\mathrm{As}_{\overline{\mathbf{C}}}^{\left(\mu, \mu^{\mathrm{op}}\right)}$ (or equivalently $\mathrm{As} \overline{\mathbf{C}}_{\overline{\mathbf{C}}, \cdot], 0)}^{(\cdot, 0)}$ provided that $\mathbf{M}$ is additive, see Remark 3.6), for classical field theory Pois $\overline{\mathbf{C}}(\{\cdot, \cdot\}, 0)$ and for linear field theory uLie $\overline{\overline{\mathbf{C}}}([\cdot, \cdot], 0)$ formalized in terms of Heisenberg Lie algebras.

\subsection{Functoriality}

Note that the field theory operad $\mathcal{P}_{\overline{\mathbf{C}}}^{\left(r_{1}, r_{2}\right)} \in \mathbf{O p}_{\mathbf{C}_{0}}(\mathbf{M})$ from Definition 3.11 depends on the choice of two kinds of data: (1) An orthogonal category $\overline{\mathbf{C}}=(\mathbf{C}, \perp)$ and (2) a bipointed single-colored operad $\mathcal{P}^{\left(r_{1}, r_{2}\right)}=\left(\mathcal{P}, r_{1}, r_{2}: I[2] \rightrightarrows U(\mathcal{P})\right)$. We will see that both of these dependencies are functorial. Recall from Definition 3.1 that orthogonal categories are the objects of the category OrthCat. The second kind of data may be arranged in terms of a category as follows.

Definition 3.14 The category of (arity 2) bipointed single-colored operads $\mathbf{O p}_{\{*\}}^{2 \mathrm{pt}}(\mathbf{M})$ has the following objects and morphisms: An object is a pair $\mathcal{P}^{\left(r_{1}, r_{2}\right)}=\left(\mathcal{P}, r_{1}, r_{2}\right.$ : $I[2] \rightrightarrows U(\mathcal{P}))$ consisting of a single-colored operad $\mathcal{P} \in \mathbf{O} \mathbf{p}_{\{*\}}(\mathbf{M})$ and a parallel pair of $\mathbf{S e q}_{\{*\}}(\mathbf{M})$-morphisms $r_{1}, r_{2}: I[2] \rightrightarrows U(\mathcal{P})$ [see (3.2) for the definition of $I[2]]$. A morphism $\phi: \mathcal{P}^{\left(r_{1}, r_{2}\right)} \rightarrow \mathcal{Q}^{\left(s_{1}, s_{2}\right)}$ is an $\mathbf{O p}_{\{*\}}(\mathbf{M})$-morphism $\phi: \mathcal{P} \rightarrow \mathcal{Q}$ that preserves the points, i.e. the diagram

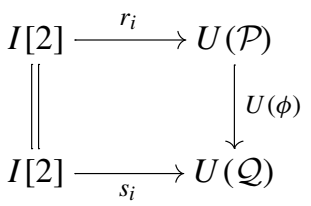

in $\operatorname{Seq}_{\{*\}}(\mathbf{M})$ commutes for $i=1,2$.

Proposition 3.15 The assignment $\left(\overline{\mathbf{C}}, \mathcal{P}^{\left(r_{1}, r_{2}\right)}\right) \longmapsto\left(\mathbf{C}_{0}, \mathcal{P}_{\overline{\mathbf{C}}}^{\left(r_{1}, r_{2}\right)}\right)$ of the field theory operads from Definition 3.11 naturally extends to a functor $\mathbf{O r t h C a t} \times \mathbf{O p}_{\{*\}}^{2 p t}(\mathbf{M}) \rightarrow$ $\mathbf{O p}(\mathbf{M})$ with values in the category of operads with varying colors (see Definition 2.10 ).

Proof For every morphism $(F, \phi):\left(\overline{\mathbf{C}}, \mathcal{P}^{\left(r_{1}, r_{2}\right)}\right) \rightarrow\left(\overline{\mathbf{D}}, \mathcal{Q}^{\left(s_{1}, s_{2}\right)}\right)$ in OrthCat $\times$ $\mathbf{O} \mathbf{p}_{\{*\}}^{2 \mathrm{pt}}(\mathbf{M})$, one can define an $\mathbf{O p}(\mathbf{M})$-morphism $\phi_{F}: \mathcal{P}_{\mathbf{C}} \rightarrow \mathcal{Q}_{\mathbf{D}}$ between the corresponding auxiliary operads from Definition 3.9. Concretely, this morphism is specified by the components 


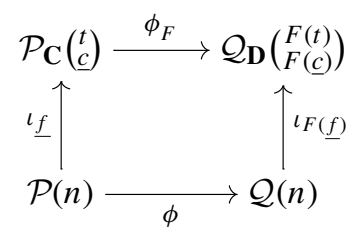

We now show that the assignment of the field theory operads is functorial too. For this we first note that one can define analogously to above a morphism $R_{\perp_{\mathbf{C}}} \rightarrow R_{\perp_{\mathbf{D}}}$ of colored sequences and one easily checks that this defines a morphism of parallel pairs in (3.19). (For this step, one uses that $F$ is an orthogonal functor and that $\phi$ preserves the points.) Because forming colimits is functorial, this defines an $\mathbf{O p}_{\mathbf{C}_{0}}(\mathbf{M})$-morphism $\mathcal{P}_{\overline{\mathbf{C}}}^{\left(r_{1}, r_{2}\right)} \rightarrow \operatorname{colim}\left(F^{*}\left(F\left(R_{\perp_{\mathbf{D}}}\right)\right) \rightrightarrows F^{*}\left(\mathcal{Q}_{\mathbf{D}}\right)\right)$ to the coequalizer of the corresponding pullback operads. (With an abuse of notation, we denoted by $F$ both the free $\mathbf{D}_{0}$-colored operad functor (2.4) and the orthogonal functor $F: \overline{\mathbf{C}} \rightarrow \overline{\mathbf{D}}$.) Notice that pullback operads arise at this point because Definition 3.11 considers colimits in the categories of operads with a fixed set of colors and not in the category $\mathbf{O p}(\mathbf{M})$. From the universal property of colimits, one obtains a canonical $\mathbf{O p}_{\mathbf{C}_{0}}(\mathbf{M})$ $\operatorname{morphism} \operatorname{colim}\left(F^{*}\left(F\left(R_{\perp_{\mathbf{D}}}\right)\right) \rightrightarrows F^{*}\left(\mathcal{Q}_{\mathbf{D}}\right)\right) \rightarrow F^{*}\left(\mathcal{Q}_{\overline{\mathbf{D}}}^{\left(s_{1}, s_{2}\right)}\right)$ to the pullback of field theory operad. The composition of the latter two morphisms defines our desired $\mathbf{O p}(\mathbf{M})$-morphism, which we denote with abuse of notation by the same symbol $\phi_{F}: \mathcal{P}_{\overline{\mathbf{C}}}^{\left(r_{1}, r_{2}\right)} \rightarrow \mathcal{Q}_{\overline{\mathbf{D}}}^{\left(s_{1}, s_{2}\right)}$ as the one for the auxiliary operads.

As a consequence of this proposition, we obtain for every morphism $(F, \phi)$ : $\left(\overline{\mathbf{C}}, \mathcal{P}^{\left(r_{1}, r_{2}\right)}\right) \rightarrow\left(\overline{\mathbf{D}}, \mathcal{Q}^{\left(s_{1}, s_{2}\right)}\right)$ in OrthCat $\times \mathbf{O p}_{\{*\}}^{2 \mathrm{pt}}(\mathbf{M})$ an $\mathbf{O p}(\mathbf{M})$-morphism $\phi_{F}:$ $\mathcal{P}_{\overline{\mathbf{C}}}^{\left(r_{1}, r_{2}\right)} \rightarrow \mathcal{Q}_{\overline{\mathbf{D}}}^{\left(s_{1}, s_{2}\right)}$ and hence by Theorems 2.15 and 3.12 an adjunction

$$
\left(\phi_{F}\right)_{!}: \mathbf{F T}\left(\overline{\mathbf{C}}, \mathcal{P}^{\left(r_{1}, r_{2}\right)}\right) \rightleftarrows \mathbf{F T}\left(\overline{\mathbf{D}}, \mathcal{Q}^{\left(s_{1}, s_{2}\right)}\right):\left(\phi_{F}\right)^{*}
$$

between the corresponding categories of field theories. From the concrete definition of $\phi_{F}$ given in the proof of Proposition 3.15 and the identification in Theorem 3.12, one observes that the right adjoint $\left(\phi_{F}\right)^{*}$ admits a very explicit description in terms of either of the two compositions in the commutative diagram

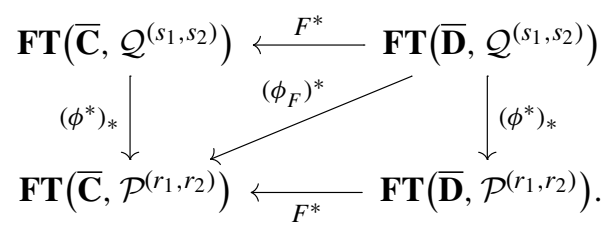

In this diagram, $F^{*}$ is the restriction to the categories of field theories of the pullback functor for functor categories

$$
F^{*}:=(-) \circ F: \mathbf{A l g}_{\mathcal{O}} \mathbf{D} \longrightarrow \mathbf{A l g}_{\mathcal{O}} \mathbf{C}
$$


for $\mathcal{O}=\mathcal{P}$ and $\mathcal{O}=\mathcal{Q}$, and $\left(\phi^{*}\right)_{*}$ is the restriction to the categories of field theories of the pushforward functor for functor categories

$$
\left(\phi^{*}\right)_{*}:=\phi^{*} \circ(-): \mathbf{A l g}_{\mathcal{Q}}{ }^{\mathbf{E}} \longrightarrow \mathbf{A l g}_{\mathcal{P}}{ }^{\mathbf{E}}
$$

for $\mathbf{E}=\mathbf{C}$ and $\mathbf{E}=\mathbf{D}$, where $\phi^{*}: \mathbf{A} \mathbf{l g}_{\mathcal{Q}} \rightarrow \mathbf{A} \mathbf{I g}_{\mathcal{P}}$ is the pullback functor corresponding to the single-colored operad morphism $\phi: \mathcal{P} \rightarrow \mathcal{Q}$.

\section{Universal constructions for field theories}

This section is concerned with analyzing in more depth the adjunctions in (3.25) and their relevance for universal constructions in field theory. Because of (3.26), this problem may be decomposed into three smaller building blocks:

1. Adjunctions induced by orthogonal functors $F: \overline{\mathbf{C}} \rightarrow \overline{\mathbf{D}}$

$$
F_{!}: \mathbf{F T}\left(\overline{\mathbf{C}}, \mathcal{P}^{\left(r_{1}, r_{2}\right)}\right) \longleftrightarrow \mathbf{F T}\left(\overline{\mathbf{D}}, \mathcal{P}^{\left(r_{1}, r_{2}\right)}\right): F^{*}
$$

2. Adjunctions induced by $\mathbf{O} \mathbf{p}_{\{*\}}^{2 \mathrm{pt}}(\mathbf{M})$-morphisms $\phi: \mathcal{P}^{\left(r_{1}, r_{2}\right)} \rightarrow \mathcal{Q}^{\left(s_{1}, s_{2}\right)}$

$$
\left(\phi^{*}\right)^{!}: \mathbf{F T}\left(\overline{\mathbf{C}}, \mathcal{P}^{\left(r_{1}, r_{2}\right)}\right) \rightleftarrows \mathbf{F T}\left(\overline{\mathbf{C}}, \mathcal{Q}^{\left(s_{1}, s_{2}\right)}\right):\left(\phi^{*}\right)_{*}
$$

3. The interplay between these two cases via the diagram of categories and functors

$$
\begin{aligned}
& \mathbf{F T}\left(\overline{\mathbf{C}}, \mathcal{Q}^{\left(s_{1}, s_{2}\right)}\right) \underset{F^{*}}{\stackrel{F_{!}}{\rightleftarrows}} \mathbf{F T}\left(\overline{\mathbf{D}}, \mathcal{Q}^{\left(s_{1}, s_{2}\right)}\right) \\
& \left(\phi^{*}\right)^{!} \uparrow\left|\downarrow\left(\phi^{*}\right)_{*} \quad F^{*} \quad\left(\phi^{*}\right) !\right| \downarrow\left(\phi^{*}\right)_{*} \\
& \mathbf{F T}\left(\overline{\mathbf{C}}, \mathcal{P}^{\left(r_{1}, r_{2}\right)}\right) \underset{F^{*}}{\stackrel{F_{!}}{\rightleftarrows}} \mathbf{F T}\left(\overline{\mathbf{D}}, \mathcal{P}^{\left(r_{1}, r_{2}\right)}\right)
\end{aligned}
$$

in which the square formed by the right adjoints commutes by (3.26) and, as a consequence of the uniqueness (up to a unique natural isomorphism) of left adjoint functors, the square formed by the left adjoints commutes up to a unique natural isomorphism.

In the following subsections, we study particular classes of examples of such adjunctions, all of which are motivated by concrete problems and constructions in field theory, and discuss their interplay. A particularly interesting example, which we will discuss later in Sect. 5, is given by an adjunction that describes the quantization of linear field theories. 


\subsection{Full orthogonal subcategories}

Recall from [8] that a full orthogonal subcategory of an orthogonal category $\overline{\mathbf{D}}=$ $\left(\mathbf{D}, \perp_{\mathbf{D}}\right)$ is a full subcategory $\mathbf{C} \subseteq \mathbf{D}$ that is endowed with the pullback orthogonality relation, i.e. $f_{1} \perp_{\mathbf{C}} f_{2}$ if and only if $f_{1} \perp_{\mathbf{D}} f_{2}$. The embedding functor $j: \mathbf{C} \rightarrow \mathbf{D}$ defines an orthogonal functor $j: \overline{\mathbf{C}} \rightarrow \overline{\mathbf{D}}$.

Proposition 4.1 Let $j: \overline{\mathbf{C}} \rightarrow \overline{\mathbf{D}}$ be a full orthogonal subcategory and $\mathcal{P}^{\left(r_{1}, r_{2}\right)} \in$ $\mathbf{O} \mathbf{p}_{\{*\}}^{2 p t}(\mathbf{M})$ a bipointed single-colored operad. Then the corresponding adjunction

$$
j !: \mathbf{F T}\left(\overline{\mathbf{C}}, \mathcal{P}^{\left(r_{1}, r_{2}\right)}\right) \rightleftarrows \mathbf{F T}\left(\overline{\mathbf{D}}, \mathcal{P}^{\left(r_{1}, r_{2}\right)}\right): j^{*}
$$

exhibits $\mathbf{F T}\left(\overline{\mathbf{C}}, \mathcal{P}^{\left(r_{1}, r_{2}\right)}\right)$ as a full coreflective subcategory of $\mathbf{F T}\left(\overline{\mathbf{D}}, \mathcal{P}^{\left(r_{1}, r_{2}\right)}\right)$, i.e. the unit $\eta: i d \rightarrow j^{*} j$ ! of this adjunction is a natural isomorphism.

Proof The proof is analogous to the corresponding one in [8] and will not be repeated.

Example 4.2 Recall the orthogonal category $\overline{\text { Loc }}$ of globally hyperbolic spacetimes from Example 3.2. Consider the full subcategory $\mathbf{L o c}_{\diamond} \subseteq$ Loc of all spacetimes whose underlying manifold is diffeomorphic to $\mathbb{R}^{m}$. Endowed with the pullback orthogonality relation, i.e. $f_{1} \perp_{\mathbf{L o c}_{\diamond}} f_{2}$ if and only if $f_{1} \perp_{\mathbf{L o c}} f_{2}$, this defines a full orthogonal subcategory $j: \overline{\mathbf{L o c}_{\diamond}} \rightarrow \overline{\mathbf{L o c}}$. The corresponding adjunction is

$$
j !: \mathbf{F T}\left(\overline{\mathbf{L o c}_{\diamond}}, \mathcal{P}^{\left(r_{1}, r_{2}\right)}\right) \rightleftarrows \mathbf{F T}\left(\overline{\mathbf{L o c}}, \mathcal{P}^{\left(r_{1}, r_{2}\right)}\right): j^{*}
$$

The right adjoint $j^{*}$ is the restriction functor which restricts field theories that are defined on all of $\overline{\mathbf{L o c}}$ to the full orthogonal subcategory $\overline{\mathbf{L o c}_{\diamond}}$ of spacetimes diffeomorphic to $\mathbb{R}^{m}$. More interestingly, the left adjoint $j$ ! is a universal extension functor which extends field theories that are only defined on $\overline{\mathbf{L O c}_{\diamond}}$ to all of $\overline{\mathbf{L O c}}$. It was shown in [8] that $j$ ! is a generalization and refinement of Fredenhagen's universal algebra construction [19-21,31].

A non-trivial application of a similar universal extension functor for quantum field theories on spacetimes with boundaries has been studied in [4]. It has been shown that the ideals of the universal extension $j_{!}(\mathfrak{B})$ of a theory $\mathfrak{B}$ that is defined only on the interior of a spacetime with boundary are related to boundary conditions.

Remark 4.3 The result in Proposition 4.1 that $j$ ! exhibits $\mathbf{F T}\left(\overline{\mathbf{C}}, \mathcal{P}^{\left(r_{1}, r_{2}\right)}\right)$ as a full coreflective subcategory of $\mathbf{F T}\left(\overline{\mathbf{D}}, \mathcal{P}^{\left(r_{1}, r_{2}\right)}\right)$ is crucial for a proper interpretation of $j$ ! as a universal extension functor and $j^{*}$ as a restriction functor in the spirit of Example 4.2. Given any field theory $\mathfrak{B} \in \mathbf{F T}\left(\overline{\mathbf{C}}, \mathcal{P}^{\left(r_{1}, r_{2}\right)}\right)$ on the full orthogonal subcategory $\overline{\mathbf{C}} \subseteq \overline{\mathbf{D}}$, one may apply the left and then the right adjoint functor in (4.4) to obtain another field theory $j^{*} j !(\mathfrak{B}) \in \mathbf{F T}\left(\overline{\mathbf{C}}, \mathcal{P}^{\left(r_{1}, r_{2}\right)}\right)$ on $\overline{\mathbf{C}} \subseteq \overline{\mathbf{D}}$. The latter is interpreted as the restriction of the universal extension of $\mathfrak{B}$. By Proposition 4.1, the unit $\eta_{\mathfrak{B}}: \mathfrak{B} \rightarrow j^{*} j$ ! $(\mathfrak{B})$ 
defines an isomorphism between these two theories, which means that $j$ ! extends field theories from $\overline{\mathbf{C}} \subseteq \overline{\mathbf{D}}$ to all of $\overline{\mathbf{D}}$ without altering their values on the subcategory $\overline{\mathbf{C}}$.

With this observation in mind, we would like to comment on existing criticisms that universal constructions, such as Fredenhagen's universal algebra or our universal extension $j_{\text {! }}$, may fail to provide a non-trivial result, see e.g. [32] and also [31]. (We would like to thank the anonymous referee for bringing this to our attention.) It is indeed true that the algebra $j_{!}(\mathfrak{B})(d) \in \mathbf{A l g}_{\mathcal{P}}$ associated with an object $d \in \mathbf{D}$ that is not in the subcategory $\mathbf{C} \subseteq \mathbf{D}$, i.e. $d \notin \mathbf{C}$, might be trivial. However, for every non-trivial $\mathfrak{B} \in \mathbf{F T}\left(\overline{\mathbf{C}}, \mathcal{P}^{\left(r_{1}, r_{2}\right)}\right)$, the universally extended field theory $j_{!}(\mathfrak{B}) \in \mathbf{F T}\left(\overline{\mathbf{D}}, \mathcal{P}^{\left(r_{1}, r_{2}\right)}\right)$ as a whole is non-trivial because, as we explained above, its restriction $j^{*} j$ ! $(\mathfrak{B})$ to $\overline{\mathbf{C}}$ is isomorphic to the input $\mathfrak{B}$ of the construction. We expect that one can construct physical examples of such theories that are non-trivial on simple spacetimes in $\overline{\mathbf{C}}$, but might be trivial on certain complicated spacetimes in $\overline{\mathbf{D}}$, by considering field equations that admit only local solutions.

An interesting application of the class of adjunctions in (4.4) is that they allow us to formalize a kind of local-to-global (i.e. descent) condition for field theories. Given a field theory $\mathfrak{A} \in \mathbf{F T}\left(\overline{\mathbf{D}}, \mathcal{P}^{\left(r_{1}, r_{2}\right)}\right)$ on the bigger category $\overline{\mathbf{D}}$, one may ask whether it is already completely determined by its values on the full orthogonal subcategory $\overline{\mathbf{C}} \subseteq \overline{\mathbf{D}}$. In the context of Example 4.2, this means asking if the value of a field theory on a general spacetime $M \in \overline{\mathbf{L O c}}$ is completely determined by its behavior on spacetimes diffeomorphic to $\mathbb{R}^{m}$, which is a typical question of descent. The following definition provides a formalization of this idea.

Definition 4.4 A field theory $\mathfrak{A} \in \mathbf{F T}\left(\overline{\mathbf{D}}, \mathcal{P}^{\left(r_{1}, r_{2}\right)}\right)$ on $\overline{\mathbf{D}}$ is called $j$-local if the corresponding component of the counit $\epsilon_{\mathfrak{A}}: j_{!} j^{*}(\mathfrak{A}) \rightarrow \mathfrak{A}$ is an isomorphism. The full subcategory of $j$-local field theories is denoted by $\mathbf{F T}\left(\overline{\mathbf{D}}, \mathcal{P}^{\left(r_{1}, r_{2}\right)}\right)^{j \text {-loc }} \subseteq$ $\mathbf{F T}\left(\overline{\mathbf{D}}, \mathcal{P}^{\left(r_{1}, r_{2}\right)}\right)$.

The following result, which extends earlier results from [8] to our more general framework, shows that $j$-local field theories on the bigger category $\overline{\mathbf{D}}$ may be equivalently described by field theories on the full orthogonal subcategory $\overline{\mathbf{C}} \subseteq \overline{\mathbf{D}}$.

Corollary 4.5 The adjunction (4.4) restricts to an adjoint equivalence

$$
j !: \mathbf{F T}\left(\overline{\mathbf{C}}, \mathcal{P}^{\left(r_{1}, r_{2}\right)}\right) \underset{\mathbf{F T}}{\longleftarrow}\left(\overline{\mathbf{D}}, \mathcal{P}^{\left(r_{1}, r_{2}\right)}\right)^{j-l o c}: j^{*}
$$

Proof This is an immediate consequence of Proposition 4.1.

Example 4.6 Being a powerful local-to-global property, it is in general not easy to prove that a given field theory $\mathfrak{A} \in \mathbf{F T}\left(\overline{\mathbf{D}}, \mathcal{P}^{\left(r_{1}, r_{2}\right)}\right)$ on $\overline{\mathbf{D}}$ is $j$-local for some full orthogonal subcategory embedding $j: \overline{\mathbf{C}} \rightarrow \overline{\mathbf{D}}$. Positive results are known for the usual Klein-Gordon quantum field theory and $j: \overline{\mathbf{L o c}_{\diamond}} \rightarrow \overline{\mathbf{L o c}}$, see [31] and [8, Section 5]. We expect that this proof can be adapted to cover all vector bundle valued linear quantum field theories in the sense of [1-3]. 


\subsection{Orthogonal localizations}

Recall from [8] that the orthogonal localization of an orthogonal category $\overline{\mathbf{C}}$ at a subset $W \subseteq$ Mor $\mathbf{C}$ of the set of morphisms is given by the localized category $\mathbf{C}\left[W^{-1}\right]$ endowed with the pushforward orthogonality relation $\perp_{\mathbf{C}\left[W^{-1}\right]}:=L_{*}\left(\perp_{\mathbf{C}}\right)$ along the localization functor $L: \mathbf{C} \rightarrow \mathbf{C}\left[W^{-1}\right]$, i.e. $\perp_{\mathbf{C}\left[W^{-1}\right]}$ is the smallest orthogonality relation such that $L\left(f_{1}\right) \perp_{\mathbf{C}\left[W^{-1}\right]} L\left(f_{2}\right)$ for all $f_{1} \perp_{\mathbf{C}} f_{2}$. The localization functor defines an orthogonal functor $L: \overline{\mathbf{C}} \rightarrow \overline{\mathbf{C}\left[W^{-1}\right]}$.

Proposition 4.7 Let $L: \overline{\mathbf{C}} \rightarrow \overline{\mathbf{C}\left[W^{-1}\right]}$ be an orthogonal localization and $\mathcal{P}^{\left(r_{1}, r_{2}\right)} \in$ $\mathbf{O} \mathbf{p}_{\{*\}}^{2 p t}(\mathbf{M})$ a bipointed single-colored operad. Then the corresponding adjunction

$$
L_{!}: \mathbf{F T}\left(\overline{\mathbf{C}}, \mathcal{P}^{\left(r_{1}, r_{2}\right)}\right) \rightleftarrows \mathbf{F T}\left(\overline{\mathbf{C}\left[W^{-1}\right]}, \mathcal{P}^{\left(r_{1}, r_{2}\right)}\right): L^{*}
$$

exhibits $\mathbf{F T}\left(\overline{\mathbf{C}\left[W^{-1}\right]}, \mathcal{P}^{\left(r_{1}, r_{2}\right)}\right)$ as a full reflective subcategory of $\mathbf{F T}\left(\overline{\mathbf{C}}, \mathcal{P}^{\left(r_{1}, r_{2}\right)}\right)$, i.e. the counit $\epsilon: L ! L^{*} \rightarrow$ id of this adjunction is a natural isomorphism.

Proof The proof is analogous to the corresponding one in [8] and will not be repeated.

Let us now explain in some detail the relationship between the adjunction (4.7) and a suitable generalization of the 'time-slice axiom' that we shall call $W$-constancy.

Definition 4.8 A field theory $\mathfrak{A} \in \mathbf{F T}\left(\overline{\mathbf{C}}, \mathcal{P}^{\left(r_{1}, r_{2}\right)}\right)$ is called $W$-constant if the $\mathbf{A l g}_{\mathcal{P}^{-}}$ morphism $\mathfrak{A}(f): \mathfrak{A}(c) \rightarrow \mathfrak{A}\left(c^{\prime}\right)$ is an isomorphism, for all $\left(f: c \rightarrow c^{\prime}\right) \in W$. The full subcategory of $W$-constant field theories is denoted by $\mathbf{F T}\left(\overline{\mathbf{C}}, \mathcal{P}^{\left(r_{1}, r_{2}\right)}\right)^{W \text {-const } \subseteq}$ $\mathbf{F T}\left(\overline{\mathbf{C}}, \mathcal{P}^{\left(r_{1}, r_{2}\right)}\right)$.

Proposition 4.9 The adjunction (4.7) restricts to an adjoint equivalence

$$
L_{!}: \mathbf{F T}\left(\overline{\mathbf{C}}, \mathcal{P}^{\left(r_{1}, r_{2}\right)}\right)^{W \text {-const }} \underset{\sim}{\longrightarrow T}\left(\overline{\mathbf{C}\left[W^{-1}\right]}, \mathcal{P}^{\left(r_{1}, r_{2}\right)}\right): L^{*}
$$

As a consequence, a field theory $\mathfrak{A} \in \mathbf{F T}\left(\overline{\mathbf{C}}, \mathcal{P}^{\left(r_{1}, r_{2}\right)}\right)$ is $W$-constant if and only if the corresponding component $\eta_{\mathfrak{A}}: \mathfrak{A} \rightarrow L^{*} L_{!}(\mathfrak{A})$ of the unit of the adjunction (4.7) is an isomorphism.

Proof By Proposition 4.7, we already know that the right adjoint functor $L^{*}$ is fully faithful, and hence it remains to prove that its essential image is $\mathbf{F T}\left(\overline{\mathbf{C}}, \mathcal{P}^{\left(r_{1}, r_{2}\right)}\right)^{W \text {-const }}$. The image of $L^{*}$ lies in $\mathbf{F T}\left(\overline{\mathbf{C}}, \mathcal{P}^{\left(r_{1}, r_{2}\right)}\right)^{W \text {-const }}$ because, for every $\mathfrak{B} \in \mathbf{F T}\left(\overline{\mathbf{C}\left[W^{-1}\right]}\right.$, $\left.\mathcal{P}^{\left(r_{1}, r_{2}\right)}\right)$, the field theory $L^{*}(\mathfrak{B})$ is $W$-constant since $L^{*}=(-) \circ L$ is given by restricting the pullback functor for functor categories and the localization functor $L$ : $\mathbf{C} \rightarrow \mathbf{C}\left[W^{-1}\right]$ maps morphisms in $W$ to isomorphisms. To prove essential surjectivity, let $\mathfrak{A} \in \mathbf{F T}\left(\overline{\mathbf{C}}, \mathcal{P}^{\left(r_{1}, r_{2}\right)}\right)^{W \text {-const }}$ and consider its underlying functor $\mathfrak{A}: \mathbf{C} \rightarrow \mathbf{A l g}_{\mathcal{P}}$. By definition of localization, there exists a functor $\mathfrak{B}: \mathbf{C}\left[W^{-1}\right] \rightarrow \mathbf{A l g}_{\mathcal{P}}$ and a 
natural isomorphism $\mathfrak{A} \cong L^{*}(\mathfrak{B})$. Using that the orthogonality relation $\perp_{\mathbf{C}\left[W^{-1}\right]}$ is generated by $L\left(f_{1}\right) \perp_{\mathbf{C}\left[W^{-1}\right]} L\left(f_{2}\right)$, for all $f_{1} \perp_{\mathbf{C}} f_{2}$, one easily checks that $\mathfrak{B} \in \mathbf{F T}\left(\overline{\mathbf{C}\left[W^{-1}\right]}, \mathcal{P}^{\left(r_{1}, r_{2}\right)}\right)$.

Example 4.10 Recall the orthogonal category $\overline{\text { Loc }}$ of globally hyperbolic spacetimes from Example 3.2 and consider the subset $W \subseteq$ Mor Loc of all Cauchy morphisms, i.e. morphisms $f: M \rightarrow M^{\prime}$ whose image $f(M) \subseteq M^{\prime}$ contains a Cauchy surface of $M^{\prime}$. In this case, $W$-constant field theories are precisely field theories that satisfy the usual time-slice axiom with respect to all Cauchy morphisms. As a consequence of Proposition 4.9, such field theories can be described equivalently as field theories on the orthogonal localization $\overline{\mathbf{L o c}\left[W^{-1}\right]}$ of $\overline{\mathbf{L o c}}$ at all Cauchy morphisms $W$. This alternative point of view comes together with an adjunction

$$
L_{!}: \mathbf{F T}\left(\overline{\mathbf{L o c}}, \mathcal{P}^{\left(r_{1}, r_{2}\right)}\right) \longleftrightarrow \mathbf{F T}\left(\overline{\operatorname{Loc}\left[W^{-1}\right]}, \mathcal{P}^{\left(r_{1}, r_{2}\right)}\right): L^{*}
$$

which allows us to detect $W$-constancy of a field theory $\mathfrak{A} \in \mathbf{F T}\left(\overline{\mathbf{L o c}}, \mathcal{P}^{\left(r_{1}, r_{2}\right)}\right)$ by testing whether the unit $\eta_{\mathfrak{A}}: \mathfrak{A} \rightarrow L^{*} L_{!}(\mathfrak{A})$ is an isomorphism.

The right adjoint $L^{*}$ of the adjunction (4.9) can be interpreted as the functor that forgets that a field theory $\mathfrak{B} \in \mathbf{F T}\left(\overline{\mathbf{L o c}\left[W^{-1}\right]}, \mathcal{P}^{\left(r_{1}, r_{2}\right)}\right)$ satisfies the time-slice axiom. More interestingly, the left adjoint $L$ ! assigns to a field theory $\mathfrak{A} \in \mathbf{F T}\left(\overline{\mathbf{L o c}}, \mathcal{P}^{\left(r_{1}, r_{2}\right)}\right)$ that does not necessarily satisfy the time-slice axiom a theory that does. Hence, one may call the left adjoint functor $L$ ! a 'time-slicification' functor. Notice that the result in Proposition 4.7 that $L^{*}$ exhibits $\mathbf{F T}\left(\overline{\operatorname{Loc}\left[W^{-1}\right]}, \mathcal{P}^{\left(r_{1}, r_{2}\right)}\right)$ as a full reflective subcategory of FT $\left(\overline{\mathbf{L o c}}, \mathcal{P}^{\left(r_{1}, r_{2}\right)}\right)$ has a concrete meaning. The isomorphisms $\epsilon_{\mathfrak{B}}: L ! L^{*}(\mathfrak{B}) \rightarrow \mathfrak{B}$ given by the counit say that time-slicification does not alter those field theories that already do satisfy the time-slice axiom, which is of course a very reasonable property.

To conclude we consider the following example in order to show that our 'timeslicification' functor does not generically produce trivial field theories. Let $\mathfrak{B}=$ $\mathfrak{A} / \mathcal{I} \in \mathbf{F T}\left(\overline{\operatorname{Loc}\left[W^{-1}\right]}, \mathcal{P}^{\left(r_{1}, r_{2}\right)}\right)$ be a field theory satisfying the time-slice axiom that is obtained by quotienting out an equation of motion ideal $\mathcal{I}$ of an 'off-shell' field theory $\mathfrak{A}$. More formally, this means that $L^{*}(\mathfrak{B}) \in \mathbf{F T}\left(\overline{\mathbf{L o c}}, \mathcal{P}^{\left(r_{1}, r_{2}\right)}\right)$ is given by a coequalizer

$$
F(\mathcal{I}) \Longrightarrow \mathfrak{A}--\rightarrow L^{*}(\mathfrak{B})
$$

in the category $\mathbf{F T}\left(\overline{\mathbf{L o c}}, \mathcal{P}^{\left(r_{1}, r_{2}\right)}\right)$, where $F(\mathcal{I})$ is the freely-generated field theory of the equation of motion ideal $\mathcal{I}$ and the two maps are given extending via the freeforget adjunction $F \dashv U$ the inclusion $\mathcal{I} \hookrightarrow U(\mathfrak{A})$ and the zero map $0: \mathcal{I} \rightarrow U(\mathfrak{A})$ to $F(\mathcal{I})$. The 'off-shell' field theory $\mathfrak{A} \in \mathbf{F T}\left(\overline{\mathbf{L o c}}, \mathcal{P}^{\left(r_{1}, r_{2}\right)}\right)$ is of course not assumed to satisfy the time-slice axiom. Because the 'time-slicification' functor $L_{!}$is left adjoint it preserves colimits and hence 


$$
L_{!} F(\mathcal{I}) \Longrightarrow L_{!}(\mathfrak{A})--\rightarrow L_{!} L^{*}(\mathfrak{B}) \stackrel{\epsilon_{\mathfrak{B}}}{\cong} \mathfrak{B}
$$

is a coequalizer in FT( $\left.\overline{\mathbf{L o c}\left[W^{-1}\right]}, \mathcal{P}^{\left(r_{1}, r_{2}\right)}\right)$, where we used Proposition 4.7 for the last arrow. We see that our field theory $\mathfrak{B}=\mathfrak{A} / \mathcal{I}$ may be equivalently presented as a quotient of the 'time-slicification' $L_{!}(\mathfrak{A})$ of the 'off-shell' field theory $\mathfrak{A}$. In other words, every 'on-shell' quotient of $\mathfrak{A}$ can be presented as a quotient of $L_{!}(\mathfrak{A})$, hence $L_{!}(\mathfrak{A})$ must be non-trivial provided that $\mathfrak{A}$ admits non-trivial 'on-shell' quotients.

\subsection{Change of bipointed single-colored operad}

Our third class of examples are adjunctions that correspond to morphisms $\phi$ : $\mathcal{P}^{\left(r_{1}, r_{2}\right)} \rightarrow \mathcal{Q}^{\left(s_{1}, s_{2}\right)}$ of bipointed single-colored operads, i.e.

$$
\left(\phi^{*}\right)^{!}: \mathbf{F T}\left(\overline{\mathbf{C}}, \mathcal{P}^{\left(r_{1}, r_{2}\right)}\right) \rightleftarrows \mathbf{F T}\left(\overline{\mathbf{C}}, \mathcal{Q}^{\left(s_{1}, s_{2}\right)}\right):\left(\phi^{*}\right)_{*}
$$

Let us stress that these adjunctions are conceptually very different to the ones we studied in the previous two subsections because they change the type of field theories and not the orthogonal category on which field theories are defined. In particular, such adjunctions can not be formulated within the original operadic framework for algebraic quantum field theory developed in [8] as they crucially rely on the more flexible definition 3.11 of field theory operads. In Sect. 5, we study an interesting example given by an adjunction that describes the quantization of linear field theories.

We observe the following preservation results for $j$-local field theories (see Definition 4.4) and for $W$-constant field theories (see Definition 4.8) under the adjunctions (4.12).

Proposition 4.11 Let $\phi: \mathcal{P}^{\left(r_{1}, r_{2}\right)} \rightarrow \mathcal{Q}^{\left(s_{1}, s_{2}\right)}$ be an $\mathbf{O p}_{\{*\}}^{2 p t}(\mathbf{M})$-morphism, $j: \overline{\mathbf{C}} \rightarrow \overline{\mathbf{D}}$ a full orthogonal subcategory and $W \subseteq$ Mor $\mathbf{C}$ a subset.

(a) The left adjoint functor $\left(\phi^{*}\right)^{!}: \mathbf{F T}\left(\overline{\mathbf{D}}, \mathcal{P}^{\left(r_{1}, r_{2}\right)}\right) \rightarrow \mathbf{F T}\left(\overline{\mathbf{D}}, \mathcal{Q}^{\left(s_{1}, s_{2}\right)}\right)$ preserves $j$-local field theories.

(b) The right adjoint functor $\left(\phi^{*}\right)_{*}: \mathbf{F T}\left(\overline{\mathbf{C}}, \mathcal{Q}^{\left(s_{1}, s_{2}\right)}\right) \rightarrow \mathbf{F T}\left(\overline{\mathbf{C}}, \mathcal{P}^{\left(r_{1}, r_{2}\right)}\right)$ preserves $W$-constant field theories.

Proof Item (a): Let $\mathfrak{A} \in \mathbf{F T}\left(\overline{\mathbf{D}}, \mathcal{P}^{\left(r_{1}, r_{2}\right)}\right)^{j \text {-loc }}$ be any $j$-local field theory of type $\mathcal{P}^{\left(r_{1}, r_{2}\right)}$, i.e. $\epsilon_{\mathfrak{A}}: j ! j^{*}(\mathfrak{A}) \rightarrow \mathfrak{A}$ is an isomorphism. The claim is that the field theory $\left(\phi^{*}\right)^{!}(\mathfrak{A}) \in$ $\mathbf{F T}\left(\overline{\mathbf{D}}, \mathcal{Q}^{\left(s_{1}, s_{2}\right)}\right)$ of type $\mathcal{Q}^{\left(s_{1}, s_{2}\right)}$ is $j$-local as well, i.e. $\epsilon_{\left(\phi^{*}\right)^{!}(\mathfrak{A})}: j_{!} j^{*}\left(\phi^{*}\right)^{!}(\mathfrak{A}) \rightarrow$ $\left(\phi^{*}\right) !(\mathfrak{A})$ is an isomorphism. This follows from the commutative diagram 


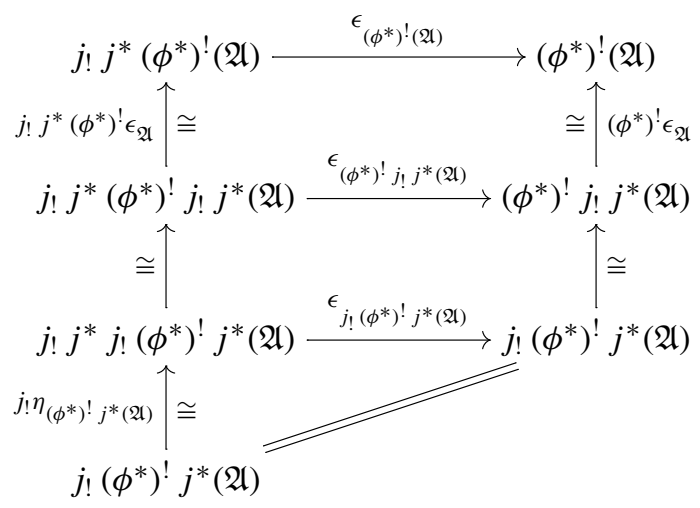

where isomorphisms are indicated by $\cong$. In more detail, the top square commutes by naturality of the counit and the vertical arrows are isomorphisms because $\mathfrak{A}$ is $j$-local. The middle square commutes because of (4.3). The bottom triangle is the triangle identity for the adjunction and the unit (vertical arrow) is an isomorphism because of Proposition 4.1. Item $(b)$ : This is immediate because $\left(\phi^{*}\right)_{*}=\phi^{*} \circ(-)$ is given by restricting the pushforward functor for functor categories and every functor $\phi^{*}$ preserves isomorphisms.

Let us emphasize that the result in Proposition 4.11 is asymmetric in the sense that $j$-local field theories are preserved by the left adjoints $\left(\phi^{*}\right)^{!}$and $W$-constant field theories are preserved by the right adjoints $\left(\phi^{*}\right)_{*}$. The opposite preservation properties do not hold true in general; however, we would like to note the following special case in which there exists a further preservation result. This will become relevant in Sect. 5 below.

Proposition 4.12 Let $\phi: \mathcal{P}^{\left(r_{1}, r_{2}\right)} \rightarrow \mathcal{Q}^{\left(s_{1}, s_{2}\right)}$ be an $\mathbf{O p}_{\{*\}}^{2 p t}(\mathbf{M})$-morphism and $W \subseteq$ Mor $\mathbf{C}$ a subset. Suppose that the left adjoint functor $\left(\phi^{*}\right)^{!}: \mathbf{F T}\left(\overline{\mathbf{C}}, \mathcal{P}^{\left(r_{1}, r_{2}\right)}\right) \rightarrow$ $\mathbf{F T}\left(\overline{\mathbf{C}}, \mathcal{Q}^{\left(s_{1}, s_{2}\right)}\right)$ is (naturally isomorphic to) the restriction to the categories of field theories of the pushforward functor for functor categories

$$
\left(\phi_{!}\right)_{*}:=\phi_{!} \circ(-): \mathbf{A l g}_{\mathcal{P}} \mathbf{C} \longrightarrow \mathbf{A l g}_{\mathcal{Q}} \mathbf{C},
$$

where the adjunction $\phi_{!}: \mathbf{A l g}_{\mathcal{P}} \rightleftarrows \mathbf{A l g}_{\mathcal{Q}}: \phi^{*}$ corresponds to the single-colored operad morphism $\phi: \mathcal{P} \rightarrow \mathcal{Q}$. Then the left adjoint functor $\left(\phi^{*}\right)^{!}: \mathbf{F T}\left(\overline{\mathbf{C}}, \mathcal{P}^{\left(r_{1}, r_{2}\right)}\right) \rightarrow$ $\mathbf{F T}\left(\overline{\mathbf{C}}, \mathcal{Q}^{\left(s_{1}, s_{2}\right)}\right)$ preserves $W$-constant field theories.

Proof This is immediate because by hypothesis there is a natural isomorphism $\left(\phi^{*}\right)^{!} \cong$

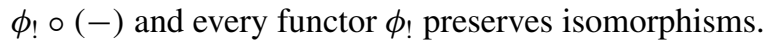

We conclude this section with a technical lemma that provides a criterion to detect whether the hypotheses of Proposition 4.12 are fulfilled. Recall from Definition 3.11 that there exists a natural projection $\mathbf{O p}_{\mathbf{C}_{0}}(\mathbf{M})$-morphism $\pi: \mathcal{P}_{\mathbf{C}} \rightarrow \mathcal{P}_{\overline{\mathbf{C}}}^{\left(r_{1}, r_{2}\right)}$ from our auxiliary operads to the field theory operads. Given any $\mathbf{O p}_{\{*\}}^{2 \mathrm{pt}}(\mathbf{M})$-morphism $\phi: \mathcal{P}^{\left(r_{1}, r_{2}\right)} \rightarrow \mathcal{Q}^{\left(s_{1}, s_{2}\right)}$, this yields the square of adjunctions 


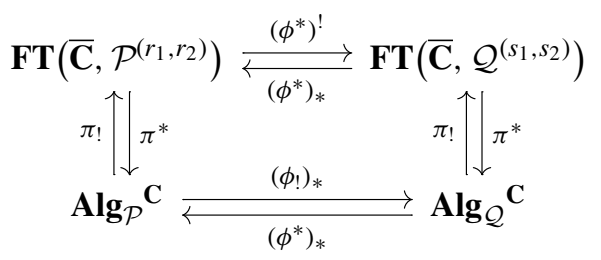

in which the square formed by the right adjoints commutes, i.e. $\left(\phi^{*}\right)_{*} \pi^{*}=\pi^{*}\left(\phi^{*}\right)_{*}$, and hence the square formed by the left adjoints commutes (up to a unique natural isomorphism), i.e. $\left(\phi^{*}\right)^{!} \pi_{!} \cong \pi_{!}\left(\phi_{!}\right)_{*}$. Notice that the vertical adjunctions exhibit the field theory categories as full reflective subcategories of the functor categories. An immediate consequence is the following

Lemma 4.13 If the functor $\left(\phi_{!}\right)_{*} \pi^{*}: \mathbf{F T}\left(\overline{\mathbf{C}}, \mathcal{P}^{\left(r_{1}, r_{2}\right)}\right) \rightarrow \operatorname{Alg}_{\mathcal{Q}} \mathbf{C}$ factors through the full reflective subcategory $\mathbf{F T}\left(\overline{\mathbf{C}}, \mathcal{Q}^{\left(s_{1}, s_{2}\right)}\right) \subseteq \operatorname{Alg}_{\mathcal{Q}} \mathbf{C}$, then the left adjoint $\left(\phi^{*}\right) !$ $\mathbf{F T}\left(\overline{\mathbf{C}}, \mathcal{P}^{\left(r_{1}, r_{2}\right)}\right) \rightarrow \mathbf{F T}\left(\overline{\mathbf{C}}, \mathcal{Q}^{\left(s_{1}, s_{2}\right)}\right)$ is (naturally isomorphic to) the restriction to the categories of field theories of the pushforward functor $\left(\phi_{!}\right)_{*}: \mathbf{A l g}_{\mathcal{P}} \mathbf{C} \rightarrow \mathbf{A l g}_{\mathcal{Q}} \mathbf{C}$.

\section{Linear quantization adjunction}

Throughout this section we assume that the underlying bicomplete closed symmetric monoidal category $\mathbf{M}$ is additive. Recalling Example 3.5 and also Remark 3.6, we define the category of quantum field theories on an orthogonal category $\overline{\mathbf{C}}$ by

$$
\operatorname{QFT}(\overline{\mathbf{C}}):=\mathbf{F T}\left(\overline{\mathbf{C}}, \mathrm{As}^{([\cdot, \cdot], 0)}\right) .
$$

Recalling further Example 3.8, we define the category of linear field theories on $\overline{\mathbf{C}}$ by

$$
\mathbf{L F T}(\overline{\mathbf{C}}):=\mathbf{F T}\left(\overline{\mathbf{C}}, \text { uLie }^{([\cdot, \cdot], 0)}\right) .
$$

We define an $\mathbf{O} \mathbf{p}_{\{*\}}(\mathbf{M})$-morphism

$$
\phi: \text { uLie } \longrightarrow \text { As, }\left\{\begin{aligned}
\eta \longmapsto \eta, \\
{[\cdot, \cdot] \longmapsto \mu-\mu^{\text {op }}, }
\end{aligned}\right.
$$

which one can confirm is well-defined by using the relations of the associative operad (see Example 2.6) and the ones of the unital Lie operad (see Example 2.9). It is evident that $\phi:$ uLie $^{([\cdot, \cdot], 0)} \rightarrow \mathrm{As}^{([\cdot, \cdot], 0)}$ defines an $\mathbf{O p}_{\{*\}}^{2 \mathrm{pt}}(\mathbf{M})$-morphism in the sense of Definition 3.14. By (4.12) this induces an adjunction between the category of linear field theories and the category of quantum field theories, which we shall denote by

$$
\left(\phi^{*}\right)^{!}=\mathfrak{Q}_{\operatorname{lin}}: \mathbf{L F T}(\overline{\mathbf{C}}) \rightleftarrows \mathbf{Q F T}(\overline{\mathbf{C}}): \mathfrak{U}_{\operatorname{lin}}=\left(\phi^{*}\right)_{*} .
$$


The aim of this section is to study this adjunction in detail and in particular to show that the left adjoint $\mathfrak{Q}_{\text {lin }}$ admits an interpretation as a linear quantization functor.

Let us first provide an explicit description of the right adjoint functor $\mathfrak{U}_{\text {lin }}=\left(\phi^{*}\right)_{*}$. Note that the functor $\phi^{*}: \mathbf{A l g}_{\mathrm{As}} \rightarrow \mathbf{A} \mathbf{A g}_{\mathrm{uLie}}$ from associative and unital algebras to unital Lie algebras is very explicit. It assigns to any $\left(A, \mu_{A}, \eta_{A}\right) \in \mathbf{A} \mathbf{A g}_{\text {As }}$ the unital Lie algebra $\phi^{*}\left(A, \mu_{A}, \eta_{A}\right)=\left(A, \mu_{A}-\mu_{A}^{\text {op }}, \eta_{A}\right) \in \mathbf{A l g}_{\text {uLie }}$, where the Lie bracket is given by the commutator. The corresponding pushforward functor $\mathfrak{U}_{\text {lin }}=\left(\phi^{*}\right)_{*}$ : QFT $(\overline{\mathbf{C}}) \rightarrow \operatorname{LFT}(\overline{\mathbf{C}})$ carries out this construction object-wise on C. Concretely, for $\left(\mathfrak{A}: \mathbf{C} \rightarrow \operatorname{Alg}_{\text {As }}\right) \in \mathbf{Q F T}(\overline{\mathbf{C}})$, the functor underlying $\mathfrak{U}_{\operatorname{lin}}(\mathfrak{A}) \in \mathbf{L F T}(\overline{\mathbf{C}})$ is given by $\mathfrak{U}_{\text {lin }}(\mathfrak{A})(c)=\phi^{*}(\mathfrak{A}(c)) \in \mathbf{A l g}_{\text {uLie }}$, for all $c \in \mathbf{C}$.

We now provide an explicit description of the left adjoint functor $\mathfrak{Q}_{\text {lin }}$ in (5.4). Our strategy is to analyze the pushforward functor $\left(\phi_{!}\right)_{*}: \mathbf{A l g}_{\mathrm{uLie}} \mathbf{C} \rightarrow \mathbf{A l g}_{\mathrm{As}} \mathbf{C}$ for the functor categories and to prove that it satisfies the criterion of Lemma 4.13. As a consequence of this lemma, the restriction to the categories of field theories of the pushforward functor $\left(\phi_{!}\right)_{*}$ defines a model for the left adjoint functor $\mathfrak{Q}_{\text {lin }}$.

Let us describe first the left adjoint functor of the adjunction $\phi_{!}: \mathbf{A l g}_{\mathrm{uLie}} \rightleftarrows \mathbf{A l g}_{\text {As }}$ : $\phi^{*}$ between algebras over single-colored operads. The following construction, which we will call the unital universal enveloping algebra construction, defines a model for the left adjoint $\phi_{!}$. Let $V \in \mathbf{A} \mathbf{I g}_{\text {uLie }}$ be any unital Lie algebra, with Lie bracket $[\cdot, \cdot]: V \otimes V \rightarrow V$ and unit $\eta: I \rightarrow V$. As the first step, we form the usual tensor algebra $T^{\otimes} V:=\bigoplus_{n=0}^{\infty} V^{\otimes n} \in \mathbf{A l g}_{\text {As }}$, i.e. the free As-algebra of the underlying object $V \in \mathbf{M}$, with multiplication $\mu_{\otimes}: T^{\otimes} V \otimes T^{\otimes} V \rightarrow T^{\otimes} V$ and unit $\eta_{\otimes}: I \rightarrow T^{\otimes} V$. We then consider the two parallel $\mathbf{M}$-morphisms

$$
V \otimes V \stackrel{q_{1}:=\left(\mu_{\otimes}-\mu_{\otimes}^{\mathrm{op}}\right)\left(\iota_{1} \otimes \iota_{1}\right)}{q_{2}:=\iota_{1}[\cdot, \cdot]} T^{\otimes} V,
$$

where $\iota_{1}: V \rightarrow T^{\otimes} V$ is the inclusion into the coproduct, which compare the commutator of $T^{\otimes} V$ with the Lie bracket of $V$. We form the corresponding coequalizer

$$
T^{\otimes}(V \otimes V) \stackrel{q_{1}}{\underset{q_{2}}{\longrightarrow}} T^{\otimes} V-\stackrel{\pi}{-} \rightarrow U^{\otimes} V
$$

in $\mathbf{A l g}_{\text {As }}$ and notice that $U^{\otimes} V$ is the universal enveloping algebra of the underlying Lie algebra $(V,[\cdot, \cdot]) \in \mathbf{A} \mathbf{A g}_{\text {Lie. }}$. As the final step, we consider the two parallel Mmorphisms

$$
I \underset{s_{2}:=\pi \eta_{\otimes}}{\stackrel{s_{1}:=\pi \iota_{1} \eta}{\longrightarrow}} U^{\otimes} V
$$

which compare the unit of $V$ with the unit of $T^{\otimes} V$, and form the corresponding coequalizer 


$$
T^{\otimes}(I) \stackrel{s_{1}}{\underset{s_{2}}{\rightrightarrows}} U^{\otimes} V-\stackrel{\pi^{\prime}}{-} \rightarrow \phi_{!}(V)
$$

in $\mathbf{A l g}_{\text {As }}$. All of these constructions are clearly functorial.

Lemma 5.1 The functor $\phi_{!}: \mathbf{A l g}_{\text {uLie }} \rightarrow \mathbf{A l g}_{\text {As }}$ described above is left adjoint to the functor $\phi^{*}: \mathbf{A l g}_{\text {As }} \rightarrow \mathbf{A l g}_{\text {uLie }}$.

Proof It is easy to construct a natural bijection $\operatorname{Hom}_{\mathrm{Alg}_{\mathrm{As}}}\left(\phi_{!}(V), A\right) \cong \operatorname{Hom}_{\mathrm{Alg}_{\mathrm{uLie}}}$ $\left(V, \phi^{*}(A)\right)$, for all $V \in \mathbf{A l g}_{\text {uLie }}$ and $A \in \mathbf{A l g}_{\text {As }}$. Concretely, given $\kappa: \phi_{!}(V) \rightarrow A$ in $\mathbf{A l g}_{\mathrm{As}}$, then $\kappa \pi^{\prime} \pi \iota_{1}: V \rightarrow \phi^{*}(A)$ defines an $\mathbf{A l g}_{\mathrm{uLie}}$-morphism. On the other hand, given $\rho: V \rightarrow \phi^{*}(A)$ in $\mathbf{A} \mathbf{l g}_{\text {uLie }}$, then the canonical extension to an $\mathbf{A} \mathbf{I g}_{\mathrm{As}}$-morphism $\rho: T^{\otimes} V \rightarrow A$ on the tensor algebra descends to the quotients in (5.5) and (5.6).

Proposition 5.2 For every linear field theory $\left(\mathfrak{B}: \mathbf{C} \rightarrow \operatorname{Alg}_{\text {uLie }}\right) \in \mathbf{L F T}(\overline{\mathbf{C}})$, the functor $\left(\phi_{!}\right)_{*}(\mathfrak{B}): \mathbf{C} \rightarrow \mathbf{A l g}_{\text {As }}$ defines a quantum field theory, i.e. $\left(\phi_{!}\right)_{*}(\mathfrak{B}) \in \mathbf{Q F T}(\overline{\mathbf{C}})$.

Proof By hypothesis, given any orthogonal pair $\left(f_{1}: c_{1} \rightarrow c\right) \perp\left(f_{2}: c_{2} \rightarrow c\right)$ in $\overline{\mathbf{C}}$, the induced Lie bracket $\left[\mathfrak{B}\left(f_{1}\right)(-), \mathfrak{B}\left(f_{2}\right)(-)\right]_{c}: \mathfrak{B}\left(c_{1}\right) \otimes \mathfrak{B}\left(c_{2}\right) \rightarrow \mathfrak{B}(c)$ is the zero map. We have to prove that the induced commutator $\left[\phi_{!} \mathfrak{B}\left(f_{1}\right)(-), \phi_{!} \mathfrak{B}\left(f_{2}\right)(-)\right]_{c}$ : $\phi_{!} \mathfrak{B}\left(c_{1}\right) \otimes \phi_{!} \mathfrak{B}\left(c_{2}\right) \rightarrow \phi_{!} \mathfrak{B}(c)$ associated to the functor $\left(\phi_{!}\right)_{*}(\mathfrak{B})=\phi_{!} \mathfrak{B}: \mathbf{C} \rightarrow$ $\mathbf{A l g}_{\text {As }}$ is the zero map too. This is an immediate consequence of our definition of the unital universal enveloping algebra [see (5.5) and (5.6)] and the fact that the commutator bracket satisfies the Leibniz rule in both entries. The latter property is used to expand the commutator of polynomials to a sum of terms containing as a factor the commutator of generators, which is identified via (5.5) with the Lie bracket.

As a consequence of Lemma 4.13, we obtain

Corollary 5.3 The restriction to the categories of field theories of $\left(\phi_{!}\right)_{*}: \mathbf{A l g}_{u L i e} \mathbf{C} \rightarrow$ $\mathbf{A l g}_{A s} \mathbf{C}$ is a model for the left adjoint functor $\mathfrak{Q}_{\text {lin }}: \mathbf{L F T}(\overline{\mathbf{C}}) \rightarrow \mathbf{Q F T}(\overline{\mathbf{C}})$ in (5.4).

Remark 5.4 Let us explain why $\mathfrak{Q}_{\text {lin }}: \operatorname{LFT}(\overline{\mathbf{C}}) \rightarrow$ QFT $(\overline{\mathbf{C}})$ deserves the name quantization functor. Suppose that $\mathfrak{B}=H \mathfrak{L} \in \mathbf{L F T}(\overline{\mathbf{C}})$ is the composition of a functor $\mathfrak{L}: \mathbf{C} \rightarrow$ PSymp to the category of presymplectic vector spaces with the Heisenberg Lie algebra functor $H: \mathbf{P S y m p} \rightarrow \mathbf{A l g}_{\text {uLie }}$ as described in Example 3.8. It is easy to check that the composition $\phi_{!} H: \mathbf{P S y m p} \rightarrow \mathbf{A l g}_{\mathrm{As}}$ of the Heisenberg Lie algebra functor and the unital universal enveloping algebra functor is naturally isomorphic to the usual (polynomial) CCR-algebra functor $\mathfrak{C} \mathfrak{C} \mathfrak{R}: \mathbf{P S y m p} \rightarrow \mathbf{A l g}_{\text {As }}$ that is used in the quantization of linear field theories, see e.g. [1-3]. In particular, we obtain a natural isomorphism $\mathfrak{Q}_{\text {lin }}(H \mathfrak{L}) \cong \mathfrak{C} \mathfrak{C} \mathfrak{R}: \mathbf{C} \rightarrow \mathbf{A l g}_{\text {As }}$, which means that our quantization prescription via $\mathfrak{Q}_{\text {lin }}$ is in this case equivalent to the ordinary CCR-algebra quantization of linear field theories.

We would like to emphasize that our linear quantization functor preserves both $j$-locality and $W$-constancy, i.e. it preserves descent and the time-slice axiom of field theories. 
Corollary 5.5 (a) Let $j: \overline{\mathbf{C}} \rightarrow \overline{\mathbf{D}}$ be a full orthogonal subcategory. Then the linear quantization functor $\mathfrak{Q}_{\text {lin }}: \mathbf{L F T}(\overline{\mathbf{D}}) \rightarrow \mathbf{Q F T}(\overline{\mathbf{D}})$ maps $j$-local linear field theories to $j$-local quantum field theories, (see Definition 4.4).

(b) Let $\overline{\mathbf{C}}$ be an orthogonal category and $W \subseteq$ Mor $\mathbf{C}$ a subset. Then the linear quantization functor $\mathfrak{Q}_{\text {lin }}: \mathbf{L F T}(\overline{\mathbf{C}}) \rightarrow \mathbf{Q F T}(\overline{\mathbf{C}})$ maps $W$-constant linear field theories to $W$-constant quantum field theories, (see Definition 4.8).

Proof Item (a) is a consequence of Proposition 4.11 and item (b) is a consequence of Proposition 4.12 and Corollary 5.3.

\section{Toward the quantization of linear gauge theories}

The techniques we developed in this paper can be refined to the case where $\mathbf{M}$ is a suitable symmetric monoidal model category. Let us recall that a model category is a category that comes equipped with three distinguished classes of morphisms-called weak equivalences, fibrations, and cofibrations-that satisfy a list of axioms going back to Quillen; see, e.g. [17] for a concise introduction. The main role is played by the weak equivalences, which introduce a consistent concept of "two things being the same" that is weaker than the usual concept of categorical isomorphism. For example, the category $\mathbf{M}=\mathbf{C h}(\mathbb{K})$ of (possibly unbounded) chain complexes of vector spaces over a field $\mathbb{K}$ may be endowed with a symmetric monoidal model category structure in which the weak equivalences are quasi-isomorphisms; see e.g. [30].

Model category theory plays an important role in the mathematical formulation of (quantum) gauge theories. In particular, the 'spaces' of fields in a gauge theory are actually higher spaces called stacks, which may be formalized within model category theory. We refer to [33] for the general framework and also to [7] for the example of Yang-Mills theory. Consequently, the observable 'algebras' in a quantum gauge theory are actually higher algebras, e.g. the differential graded algebras arising in the BRST/BV formalism. We refer to [22,23,29] for concrete constructions within the BRST/BV formalism in algebraic quantum field theory, to [9] for the relevant model categorical perspective and to [26] for a related deformation theoretic point of view.

The aim of this last section is to refine our results for the linear quantization adjunction from Sect. 5 to the framework of model category theory. This will provide a mathematically solid setup to quantize linear gauge theories to quantum gauge theories in a way that is consistent with the concept of weak equivalences. As an explicit example, we discuss the quantization of linear Chern-Simons theory on oriented surfaces. In order to simplify our analysis, we restrict ourselves to the case where $\mathbf{M}=\mathbf{C h}(\mathbb{K})$ is the symmetric monoidal model category of chain complexes of vector spaces over a field $\mathbb{K}$ of characteristic zero, e.g. $\mathbb{K}=\mathbb{C}$ or $\mathbb{K}=\mathbb{R}$. In this section, we shall freely use terminology and results from general model category theory $[17,30]$ and more specifically the model structures for colored operads and their algebras $[27,28]$. We refer to [6,9] for a more gentle presentation of how these techniques can be applied to $\mathbf{C h}(\mathbb{K})$-valued algebraic quantum field theory. 


\subsection{Model structures on field theory categories}

Our first (immediate) result is that the categories $\mathbf{F T}\left(\overline{\mathbf{C}}, \mathcal{P}^{\left(r_{1}, r_{2}\right)}\right)$ of field theories with values in $\mathbf{M}=\mathbf{C h}(\mathbb{K})$ from Definition 3.3 are model categories, i.e. there exists a consistent concept of weak equivalences for $\mathbf{C h}(\mathbb{K})$-valued field theories. Furthermore, the adjunctions in (3.25) are compatible with these model category structures in the sense that they are Quillen adjunctions.

Proposition 6.1 Let $\overline{\mathbf{C}}$ be any orthogonal category and $\mathcal{P}^{\left(r_{1}, r_{2}\right)} \in \mathbf{O p}_{\{*\}}^{2 p t}(\mathbf{C h}(\mathbb{K}))$ any bipointed single-colored operad. Define a $\mathbf{F T}\left(\overline{\mathbf{C}}, \mathcal{P}^{\left(r_{1}, r_{2}\right)}\right)$-morphism $\zeta: \mathfrak{A} \rightarrow \mathfrak{B}$ (i.e. a natural transformation of functors $\left.\mathfrak{A}, \mathfrak{B}: \mathbf{C} \rightarrow \mathbf{A l g}_{\mathcal{P}}\right)$ to be

(i) a weak equivalence if the underlying $\mathbf{C h}(\mathbb{K})$-morphism of each component $\zeta_{c}$ : $\mathfrak{A}(c) \rightarrow \mathfrak{B}(c)$ is a quasi-isomorphism,

(ii) a fibration if the underlying $\mathbf{C h}(\mathbb{K})$-morphism of each component $\zeta_{c}: \mathfrak{A}(c) \rightarrow$ $\mathfrak{B}(c)$ is degree-wise surjective, and

(iii) a cofibration if it has the left-lifting property with respect to all acyclic fibrations.

These choices define a model structure on $\mathbf{F T}\left(\overline{\mathbf{C}}, \mathcal{P}^{\left(r_{1}, r_{2}\right)}\right)$.

Proof This is a consequence of Theorem 3.12 and Hinich's results [27,28], which show that all colored operads in $\mathbf{C h}(\mathbb{K})$ are admissible for $\mathbb{K}$ a field of characteristic zero.

Proposition 6.2 Let $F: \overline{\mathbf{C}} \rightarrow \overline{\mathbf{D}}$ be any orthogonal functor and $\phi: \mathcal{P}^{\left(r_{1}, r_{2}\right)} \rightarrow \mathcal{Q}^{\left(s_{1}, s_{2}\right)}$ any $\mathbf{O p}_{\{*\}}^{2 p t}(\mathbf{C h}(\mathbb{K}))$-morphism. Then the adjunction in (3.25) is a Quillen adjunction with respect to the model structures from Proposition 6.1.

Proof This follows immediately from [27,28].

As a specific instance of the general result of Proposition 6.1, we obtain that both the category of $\mathbf{C h}(\mathbb{K})$-valued linear field theories $\mathbf{L F T}(\overline{\mathbf{C}})$ and the category of $\mathbf{C h}(\mathbb{K})$ valued quantum field theories $\mathbf{Q F T}(\overline{\mathbf{C}})$ carry a canonical model structure. In order to develop a better intuition for $\mathbf{C h}(\mathbb{K})$-valued field theories and their relation to gauge theories, let us introduce a simple example of a $\mathbf{C h}(\mathbb{K})$-valued linear field theory.

Example 6.3 (Linear Chern-Simons theory on oriented surfaces) Let us denote by $\operatorname{Man}_{2}$ the category of oriented 2-manifolds and orientation preserving open embeddings. We endow $\mathbf{M a n}_{2}$ with an orthogonality relation $\perp$ by declaring two morphisms $f_{1}: M_{1} \rightarrow M$ and $f_{2}: M_{2} \rightarrow M$ to be orthogonal, $f_{1} \perp f_{2}$, if and only if their images are disjoint, i.e. $f_{1}\left(M_{1}\right) \cap f_{2}\left(M_{2}\right)=\emptyset$. The field configurations on $M \in \mathbf{M a n}_{2}$ of Chern-Simons theory with structure group $\mathbb{R}$ are given by flat $\mathbb{R}$ connections $A \in \Omega^{1}(M)$ modulo gauge transformations. In the context of linear derived geometry, see, e.g. $[6,15]$ for more details, the flatness condition $\mathrm{d} A=0$ and the quotient by gauge transformations $A \rightarrow A+\mathrm{d} \epsilon$ are both refined to higher categorical concepts called homotopy kernels and stacky quotients. This results in a chain complex of field configurations, that is given in our example by the shifted de Rham complex 


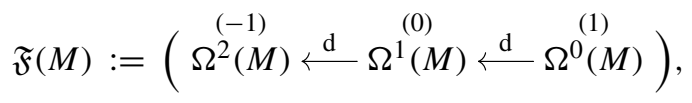

where the round brackets indicate homological degrees. Note that the zeroth homology of $\mathfrak{F}(M)$ is the usual vector space of gauge equivalence classes of flat $\mathbb{R}$-connections on $M$.

We describe linear observables on $M$ by the smooth dual of $\mathfrak{F}(M)$, which explicitly reads as

$$
\mathfrak{L}(M):=\left(\Omega_{\mathrm{c}}^{(-1)}(M) \stackrel{-\mathrm{d}}{\longleftarrow} \Omega_{\mathrm{c}}^{1}(M) \stackrel{-\mathrm{d}}{\longleftarrow} \Omega_{\mathrm{c}}^{(1)}(M)\right),
$$

where the subscript $\mathrm{c}$ denotes forms with compact support. The evaluation of observables on field configurations is given by the integration map $\int_{M}: \mathfrak{L}(M) \otimes \mathfrak{F}(M) \rightarrow \mathbb{K}$. Note that the minus signs in (6.2) are necessary for $\int_{M}$ to be a chain map. To define a presymplectic structure on $\mathfrak{L}(M)$, observe that there exists a chain map

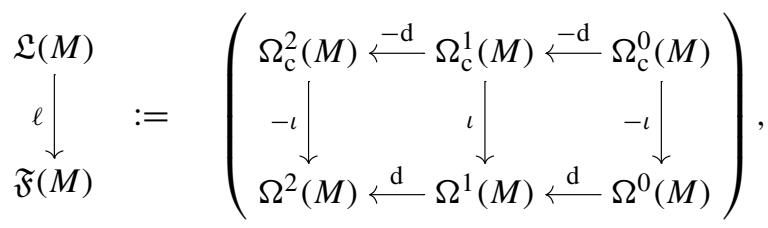

where $\iota: \Omega_{\mathrm{c}}^{p}(M) \rightarrow \Omega^{p}(M)$ denotes the inclusion of compactly supported $p$-forms into all $p$-forms. We define the chain map

$$
\omega: \quad \mathfrak{L}(M) \otimes \mathfrak{L}(M) \stackrel{\operatorname{id} \otimes \ell}{\longrightarrow} \mathfrak{L}(M) \otimes \mathfrak{F}(M) \stackrel{\int_{M}}{\longrightarrow} \mathbb{K}
$$

and note that $\omega$ is a presymplectic structure, i.e. it is graded antisymmetric.

Precisely as in Example 3.8, we can assign to the presymplectic chain complex $(\mathfrak{L}(M), \omega)$ its Heisenberg Lie algebra, which we shall denote by $\mathfrak{B}_{\mathrm{CS}}(M):=$ $\mathfrak{L}(M) \oplus \mathbb{K} \in \mathbf{A l g}_{\text {uLie. }}$. Using pushforwards of compactly supported forms along Man $_{2}$-morphisms $f: M \rightarrow N$ and observing that (6.4) are the components of a natural transformation, we can promote the assignment $M \mapsto \mathfrak{B}_{\mathrm{CS}}(M)$ to a functor $\mathfrak{B}_{\mathrm{CS}}: \mathbf{M a n}_{2} \rightarrow \mathbf{A l g}_{\mathrm{uLie}}$. Because the integration of any product of forms with disjoint support yields zero, this functor defines a $\mathbf{C h}(\mathbb{K})$-valued linear field theory $\mathfrak{B}_{\mathrm{CS}} \in \mathbf{L F T}\left(\overline{\mathbf{M a n}_{2}}\right)$ on the orthogonal category $\overline{\operatorname{Man}_{2}}$. By construction, this linear field theory describes linear Chern-Simons theory on oriented surfaces.

\subsection{Homotopical properties of linear quantization}

As a specific instance of the general result of Proposition 6.2, we obtain that the linear quantization adjunction (5.4) is a Quillen adjunction between the model categories LFT $(\overline{\mathbf{C}})$ and QFT $(\overline{\mathbf{C}})$. Using the general method of derived functors (see e.g. [17,30]), there exists a left derived linear quantization functor $\mathbb{L} \mathfrak{Q}_{\text {lin }}$ and a right derivation 
of its right adjoint $\mathbb{R} \mathfrak{U}_{\text {lin }}$. These two derived functors preserve weak equivalences and hence they are homotopically meaningful. The standard procedure to construct derived functors is to choose fibrant and cofibrant replacement functors, denoted by $R: \operatorname{QFT}(\overline{\mathbf{C}}) \rightarrow \operatorname{QFT}(\overline{\mathbf{C}})$ and $Q: \operatorname{LFT}(\overline{\mathbf{C}}) \rightarrow \operatorname{LFT}(\overline{\mathbf{C}})$, and to define the right derived functor by

$$
\mathbb{R} \mathfrak{U}_{\text {lin }}:=\mathfrak{U}_{\text {lin }} R: \mathbf{Q F T}(\overline{\mathbf{C}}) \longrightarrow \mathbf{L F T}(\overline{\mathbf{C}})
$$

and the left derived functor by

$$
\mathbb{L}_{\mathfrak{Q}_{\text {lin }}}:=\mathfrak{Q}_{\text {lin }} Q: \mathbf{L F T}(\overline{\mathbf{C}}) \longrightarrow \mathbf{Q F T}(\overline{\mathbf{C}})
$$

Note that there is some flexibility in choosing $R$ and $Q$, but different choices define naturally weakly equivalent derived functors.

For practical applications, it is crucial to find simple models for derived functors that can be computed explicitly. The goal of this subsection is to obtain such simple models for the derived functors of the linear quantization adjunction (5.4). For the right derived functor $\mathbb{R} \mathfrak{U}_{\text {lin }}$, this problem is easy to solve because every object in the model category $\mathbf{Q F T}(\overline{\mathbf{C}})$ is fibrant, hence the identity functor $R=$ id defines a fibrant replacement functor. This immediately implies

Proposition 6.4 The underived functor $\mathfrak{U}_{\text {lin }}: \mathbf{Q F T}(\overline{\mathbf{C}}) \rightarrow \mathbf{L F T}(\overline{\mathbf{C}})$ is a model for the right derived functor $\mathbb{R U}_{\text {lin }}$ in (6.5).

For the left derived functor $\mathbb{L} \mathfrak{Q}_{\text {lin }}$, i.e. the derived linear quantization functor, the situation gets more complicated because not every object in $\operatorname{LFT}(\overline{\mathbf{C}})$ is cofibrant. However, a more detailed study of $\mathfrak{Q}_{\text {lin }}$ reveals the following pleasing result.

Proposition 6.5 The underived functor $\mathfrak{Q}_{\text {lin }}: \mathbf{L F T}(\overline{\mathbf{C}}) \rightarrow \mathbf{Q F T}(\overline{\mathbf{C}})$ preserves weak equivalences. As a consequence, it is a model for the left derived functor $\mathbb{L}_{\mathfrak{Q}_{\text {lin }}}$ in (6.6).

Proof Recall from Corollary 5.3 that $\mathfrak{Q}_{\text {lin }}=\left(\phi_{!}\right)_{*}=\phi_{!} \circ(-)$ is given by postcomposing with the left adjoint functor $\phi_{!}: \mathbf{A} \mathbf{l g}_{\mathrm{uLie}} \rightarrow \mathbf{A} \mathbf{I g}_{\mathrm{As}}$. It is shown in Lemma

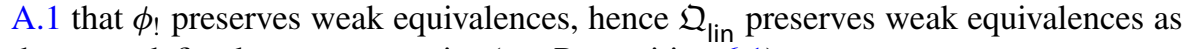
these are defined component-wise (see Proposition 6.1).

To prove the second statement, consider the natural transformation

$$
\mathbb{L} \mathfrak{Q}_{\text {lin }}=\mathfrak{Q}_{\text {lin }} Q \stackrel{\mathfrak{Q}_{\text {lin }} q}{\longrightarrow} \mathfrak{Q}_{\text {lin }}
$$

obtained by whiskering the natural weak equivalence $q: Q \rightarrow$ id corresponding to the cofibrant replacement functor $Q$. This is a natural weak equivalence because $\mathfrak{Q}_{\text {lin }}$ preserves weak equivalences, hence $\mathfrak{Q}_{\text {lin }}$ is a model for $\mathbb{L} \mathfrak{Q}_{\text {lin }}$.

Example 6.6 Let us apply these results to carry out the quantization of linear ChernSimons theory from Example 6.3. As shown in Proposition 6.5, the underived 
linear quantization functor $\mathfrak{Q}_{\text {lin }}$ provides a homotopically meaningful quantization prescription that agrees (up to weak equivalence) with any derived linear quantization functor $\mathbb{L} \mathfrak{Q}_{\text {lin }}$. Applying the functor $\mathfrak{Q}_{\text {lin }}$ to the linear Chern-Simons model $\mathfrak{B}_{\mathrm{CS}} \in \mathbf{L F T}\left(\overline{\operatorname{Man}_{2}}\right)$ from Example 6.3, we obtain the linear Chern-Simons quantum field theory $\mathfrak{A}_{\mathrm{CS}}:=\mathfrak{Q}_{\text {lin }}\left(\mathfrak{B}_{\mathrm{CS}}\right) \in \mathbf{Q F T}\left(\overline{\mathbf{M a n}_{2}}\right)$. Using the concrete description of $\mathfrak{Q}_{\text {lin }}$ given in Sect.5, we can compute explicitly the differential graded algebra $\mathfrak{A}_{\mathrm{CS}}(M) \in \mathbf{A} \mathbf{l g}_{\mathrm{As}}$ that is assigned to an oriented 2-manifold $M \in \mathbf{M a n}_{2}$. One observes that this differential graded algebra is presentable by generators and relations. The generators (6.2) are concentrated in homological degrees $-1,0$ and 1 . We shall use the intuitive 'smeared quantum field' notation to denote the generators by

$$
\widehat{C}(\chi) \in \mathfrak{A}_{\mathrm{CS}}(M)_{-1}, \quad \widehat{A}(\alpha) \in \mathfrak{A}_{\mathrm{CS}}(M)_{0}, \quad \widehat{B}(\beta) \in \mathfrak{A}_{\mathrm{CS}}(M)_{1},
$$

for all $\chi \in \Omega_{\mathrm{c}}^{2}(M), \alpha \in \Omega_{\mathrm{c}}^{1}(M)$ and $\beta \in \Omega_{\mathrm{c}}^{0}(M)$. The differential acts on these generators as

$$
\mathrm{d} \widehat{B}(\beta)=\widehat{A}(-\mathrm{d} \beta), \quad \mathrm{d} \widehat{A}(\alpha)=\widehat{C}(-\mathrm{d} \alpha), \quad \mathrm{d} \widehat{C}(\chi)=0
$$

The relations are as follows:

- $\mathbb{K}$-linearity: $\widehat{C}\left(k \chi+k^{\prime} \chi^{\prime}\right)=k \widehat{C}(\chi)+k^{\prime} \widehat{C}\left(\chi^{\prime}\right)$, for all $\chi, \chi^{\prime} \in \Omega_{\mathrm{c}}^{2}(M)$ and $k, k^{\prime} \in \mathbb{K}$, and similarly for $\widehat{A}$ and $\widehat{B}$;

- Commutation relations: The non-vanishing graded commutators are

$$
\begin{aligned}
& {\left[\widehat{A}(\alpha), \widehat{A}\left(\alpha^{\prime}\right)\right]=\omega\left(\alpha, \alpha^{\prime}\right)=\int_{M} \alpha \wedge \alpha^{\prime},} \\
& {[\widehat{C}(\chi), \widehat{B}(\beta)]=\omega(\chi, \beta)=-\int_{M} \chi \wedge \beta,} \\
& {[\widehat{B}(\beta), \widehat{C}(\chi)]=\omega(\beta, \chi)=-\int_{M} \beta \wedge \chi .}
\end{aligned}
$$

Note that the zeroth homology $H_{0}\left(\mathfrak{A}_{\mathrm{CS}}(M)\right)$ is the ordinary algebra of gauge invariant observables for quantized flat $\mathbb{R}$-connections, see e.g. [16].

\subsection{Homotopy $j$-locality and homotopy $W$-constancy}

We would like to conclude by introducing natural homotopical generalizations of the $j$-locality property (see Definition 4.4 ) and the $W$-constancy property (see Definition 4.8 ) in the context of model category theory. It will be shown that these properties are preserved by linear quantization.

Homotopy j-locality: Let $j: \overline{\mathbf{C}} \rightarrow \overline{\mathbf{D}}$ be a full orthogonal subcategory and $\mathcal{P}^{\left(r_{1}, r_{2}\right)}$ a bipointed single-colored operad. From Proposition 6.2, we obtain a Quillen adjunction $j_{!}: \mathbf{F T}\left(\overline{\mathbf{C}}, \mathcal{P}^{\left(r_{1}, r_{2}\right)}\right) \rightleftarrows \mathbf{F T}\left(\overline{\mathbf{D}}, \mathcal{P}^{\left(r_{1}, r_{2}\right)}\right): j^{*}$. For the right derived functor, we can choose again the underived functor $\mathbb{R} j^{*}:=j^{*}$, because every object in $\mathbf{F T}\left(\overline{\mathbf{D}}, \mathcal{P}^{\left(r_{1}, r_{2}\right)}\right)$ is fibrant. However, in contrast to the linear quantization functor from the previous 
subsection, the left adjoint functor $j$ ! in general does not preserve weak equivalences and hence it has to be derived $\mathbb{L}_{j !}:=j ! Q$. (See [9, Appendix A] for concrete examples illustrating this fact.) As a consequence, our previous concept of $j$-locality from Definition 4.4 has to be derived as well in order to be homotopically meaningful. In what follows, we denote by $q: Q \rightarrow$ id the natural weak equivalence corresponding to our choice of cofibrant replacement functor $Q$.

Definition 6.7 A field theory $\mathfrak{A} \in \mathbf{F T}\left(\overline{\mathbf{D}}, \mathcal{P}^{\left(r_{1}, r_{2}\right)}\right)$ is called homotopy $j$-local if the corresponding component of the derived counit

$$
\widetilde{\epsilon}_{\mathfrak{A}}: \quad j ! Q j^{*}(\mathfrak{A}) \stackrel{j ! q_{j^{*}(\mathfrak{A})}}{\longrightarrow} j ! j^{*}(\mathfrak{A}) \stackrel{\epsilon_{\mathfrak{A}}}{\longrightarrow} \mathfrak{A}
$$

is a weak equivalence in $\mathbf{F T}\left(\overline{\mathbf{D}}, \mathcal{P}^{\left(r_{1}, r_{2}\right)}\right)$.

Proposition 6.8 The linear quantization functor $\mathfrak{Q}_{\text {lin }}: \mathbf{L F T}(\overline{\mathbf{D}}) \rightarrow \operatorname{QFT}(\overline{\mathbf{D}})$ (see Proposition 6.5) maps homotopy $j$-local linear field theories to homotopy $j$-local quantum field theories.

Proof Let $\mathfrak{B} \in \mathbf{L F T}(\overline{\mathbf{D}})$ be a homotopy $j$-local linear field theory, i.e. $\widetilde{\epsilon}_{\mathfrak{B}}$ : $j ! Q j^{*}(\mathfrak{B}) \rightarrow \mathfrak{B}$ is a weak equivalence. We have to prove that the derived counit $\widetilde{\epsilon}_{\mathfrak{Q}_{\text {lin }}(\mathfrak{B})}: j ! Q j^{*} \mathfrak{Q}_{\text {lin }}(\mathfrak{B}) \rightarrow \mathfrak{Q}_{\text {lin }}(\mathfrak{B})$ corresponding to the quantum field theory $\mathfrak{Q}_{\text {lin }}(\mathfrak{B}) \in \mathbf{Q F T}(\overline{\mathbf{D}})$ is a weak equivalence too.

As a preparatory step, let us consider the commutative diagram

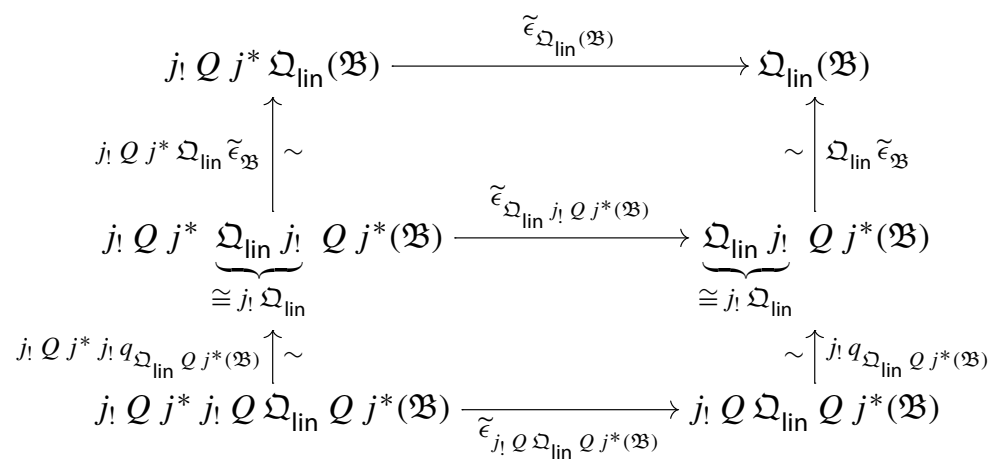

The vertical arrows in the top square are weak equivalences because $\mathfrak{B}$ is by hypothesis homotopy $j$-local. The vertical arrows in the bottom square are weak equivalences because left Quillen functors preserve cofibrant objects and weak equivalences between cofibrant objects. The natural isomorphism in the underbraces is due to (4.3). By the 2-of-3 property of weak equivalences, the top horizontal arrow is a weak equivalence (which is our claim) if and only if the bottom horizontal arrow is a weak equivalence.

Introducing $\mathfrak{A}:=\mathfrak{Q}_{\operatorname{lin}} Q j^{*}(\mathfrak{B})$, it thus remains to prove that $\tilde{\epsilon}_{j !} Q(\mathfrak{A})$ : $j_{!} Q j^{*} j_{!} Q(\mathfrak{A}) \rightarrow j_{!} Q(\mathfrak{A})$ is a weak equivalence. This follows from the 2-of-3 
property of weak equivalences and the commutative diagram

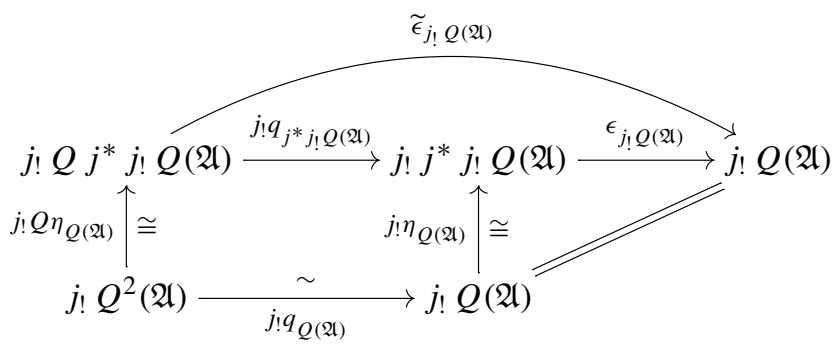

where the top triangle is just the definition of the derived counit (6.10). The vertical arrows are isomorphisms because of Proposition 4.1 and the right triangle is the triangle identity for the (non-derived) unit and counit. The bottom horizontal arrow is a weak equivalence because left Quillen functors preserve weak equivalences between cofibrant objects.

Example 6.9 Let $j: \overline{\mathbf{D i s k}_{2}} \rightarrow \overline{\mathbf{M a n}_{2}}$ be the full orthogonal subcategory of all oriented 2-manifolds $M$ that are diffeomorphic to $\mathbb{R}^{2}$. It is an interesting question whether the linear Chern-Simons quantum field theory $\mathfrak{A}_{\mathrm{CS}} \in \mathbf{Q F T}\left(\overline{\mathbf{M a n}_{2}}\right)$ from Example 6.6 is homotopy $j$-local with respect to this $j$. In particular, homotopy $j$-locality would imply that its value $\mathfrak{A}_{\mathrm{CS}}(M)$ on a topologically non-trivial oriented 2-manifold $M$ such as the torus is already encoded in the restriction $j^{*}\left(\mathfrak{A}_{\mathrm{CS}}\right) \in \mathbf{Q F T}\left(\overline{\mathbf{D i s k}_{2}}\right)$ of the quantum field theory to disks. Unfortunately, proving homotopy $j$-locality of a given theory is a complicated task and hence we can not yet provide an answer to the question whether $\mathfrak{A}_{\mathrm{CS}} \in \mathbf{Q F T}\left(\overline{\mathbf{M a n}_{2}}\right)$ is homotopy $j$-local or not. We however would like to mention that positive results are already available for simple toy-models which do not involve quantization, see [9] for details. We expect that Proposition 6.8 will be very useful for investigating homotopy $j$-locality of $\mathfrak{A}_{\mathrm{CS}}=\mathfrak{Q}_{\operatorname{lin}}\left(\mathfrak{B}_{\mathrm{CS}}\right) \in \mathbf{Q F T}\left(\overline{\mathbf{M a n}_{2}}\right)$ because it allows us to replace this question by the (slightly) simpler question whether the linear field theory $\mathfrak{B}_{\mathrm{CS}} \in \mathbf{L F T}\left(\overline{\mathbf{M a n}_{2}}\right)$ from Example 6.3 is homotopy $j$-local. We hope to come back to this issue in a future work.

Homotopy W-constancy: Let $\overline{\mathbf{C}}$ be an orthogonal category, $W \subseteq$ Mor $\mathbf{C}$ a subset and $\mathcal{P}^{\left(r_{1}, r_{2}\right)}$ a bipointed single-colored operad. Similarly to locally constant factorization algebras [15], we propose a homotopical generalization of the $W$-constancy property from Definition 4.8.

Definition 6.10 A field theory $\mathfrak{A} \in \mathbf{F T}\left(\overline{\mathbf{C}}, \mathcal{P}^{\left(r_{1}, r_{2}\right)}\right)$ is called homotopy $W$-constant if the $\mathbf{C h}(\mathbb{K})$-morphism underlying the $\mathbf{A l g}_{\mathcal{P}}$-morphism $\mathfrak{A}(f): \mathfrak{A}(c) \rightarrow \mathfrak{A}\left(c^{\prime}\right)$ is a quasi-isomorphism for all $\left(f: c \rightarrow c^{\prime}\right) \in W$.

Proposition 6.11 The linear quantization functor $\mathfrak{Q}_{\text {lin }}: \mathbf{L F T}(\overline{\mathbf{C}}) \rightarrow \mathbf{Q F T}(\overline{\mathbf{C}})$ (see Proposition 6.5) maps homotopy $W$-constant linear field theories to homotopy $W$ constant quantum field theories.

Proof Recall from Corollary 5.3 that $\mathfrak{Q}_{\text {lin }}=\left(\phi_{!}\right)_{*}=\phi_{!} \circ(-)$ is given by postcomposing with the left adjoint functor $\phi_{!}: \mathbf{A l g}_{\mathrm{uLie}} \rightarrow \mathbf{A l g}_{\mathrm{As}}$. By Lemma A.1, the 
latter preserves weak equivalences and hence it preserves the homotopy $W$-constancy property.

Example 6.12 It is easy to prove that the linear Chern-Simons model $\mathfrak{B}_{\mathrm{CS}} \in$ LFT $\left(\overline{\operatorname{Man}_{2}}\right)$ from Example 6.3 is homotopy $W$-constant for $W \subseteq \operatorname{Mor}_{\mathbf{M a n}_{2}}$ the subset of all isotopy equivalences $f: M \rightarrow M^{\prime}$. The chain map underlying $\mathfrak{B}_{\mathrm{CS}}(f): \mathfrak{B}_{\mathrm{CS}}(M) \rightarrow \mathfrak{B}_{\mathrm{CS}}\left(M^{\prime}\right)$ is given by

$$
f_{*} \oplus \mathrm{id}: \mathfrak{L}(M) \oplus \mathbb{K} \longrightarrow \mathfrak{L}\left(M^{\prime}\right) \oplus \mathbb{K}
$$

where $\mathfrak{L}(M)$ and $\mathfrak{L}\left(M^{\prime}\right)$ are (up to a global minus sign) shifted compactly supported de Rham complexes (see (6.2)) and $f_{*}$ is given by degree-wise pushforward of compactly supported forms. For $f: M \rightarrow M^{\prime}$ an isotopy equivalence, this map is a quasi-isomorphism because compactly supported de Rham cohomology is invariant under isotopies. Together with Proposition 6.11, this implies that the linear ChernSimons quantum field theory $\mathfrak{A}_{\mathrm{CS}}=\mathfrak{Q}_{\text {lin }}\left(\mathfrak{B}_{\mathrm{CS}}\right) \in \mathbf{Q F T}\left(\overline{\mathbf{M a n}_{2}}\right)$ from Example 6.6 is homotopy $W$-constant too.

Acknowledgements We would like to thank the anonymous referee for useful comments that helped us to improve this manuscript. We also would like to thank Marco Benini and Lukas Woike for useful discussions. S.B. is supported by a Ph.D. scholarship of the Royal Society (UK). A.S. gratefully acknowledges the financial support of the Royal Society (UK) through a Royal Society University Research Fellowship, a Research Grant and an Enhancement Award (Grant Nos. UF150099, RG160517, RGF $\backslash \mathrm{EA} \backslash 180270$ ).

\section{Compliance with ethical standards}

Conflict of interest On behalf of all authors, the corresponding author states that there is no conflict of interest.

Open Access This article is distributed under the terms of the Creative Commons Attribution 4.0 International License (http://creativecommons.org/licenses/by/4.0/), which permits unrestricted use, distribution, and reproduction in any medium, provided you give appropriate credit to the original author(s) and the source, provide a link to the Creative Commons license, and indicate if changes were made.

\section{A Technical details for Sect. 6.2}

In this appendix we let $\mathbf{M}=\mathbf{C h}(\mathbb{K})$ be the symmetric monoidal model category of chain complexes of vector spaces over a field $\mathbb{K}$ of characteristic zero. Recall the unital universal enveloping algebra functor $\phi_{!}: \mathbf{A l g}_{\text {uLie }} \rightarrow \mathbf{A l g}_{\text {As }}$ from Lemma 5.1. With Proposition 6.1 we see that the categories $\mathbf{A} \mathbf{I g}_{\mathrm{uLie}}$ and $\mathbf{A} \mathbf{I g}_{\mathrm{As}}$ carry a canonical model structure in which a morphism is a weak equivalence if the underlying $\mathbf{C h}(\mathbb{K})$ morphism is a quasi-isomorphism.

Lemma A.1 The functor $\phi_{!}: \mathbf{A l g}_{u L i e} \rightarrow \mathbf{A l g}_{\text {As }}$ preserves weak equivalences.

Proof As a preparation for the proof, we have to revisit the unital universal enveloping algebra construction from (5.5) and (5.6) for the category $\mathbf{C h}(\mathbb{K})$ of chain complexes. By definition, the unital universal enveloping algebra $\phi_{!}(V) \in \mathbf{A l g}_{\text {As }}$ of a unital Lie 
$\operatorname{algebra}(V,[\cdot, \cdot], \eta) \in \mathbf{A l g}_{\mathrm{uLie}}$ is the differential graded algebra presented by

$$
\phi_{!}(V)=T^{\otimes} V / \mathcal{I}
$$

where $\mathcal{I} \subseteq T^{\otimes} V$ is the differential graded ideal generated by the relations

$$
v_{1} \otimes v_{2}-(-1)^{\left|v_{1}\right|\left|v_{2}\right|} v_{2} \otimes v_{1}=\left[v_{1}, v_{2}\right], \quad \mathbb{1}_{\otimes}=\mathbb{1}
$$

for all homogeneous elements $v_{1}, v_{2} \in V$. Here $\mathbb{1}_{\otimes}=\eta_{\otimes}(1)$ denotes the unit element of the tensor algebra $T^{\otimes} V=\bigoplus_{m=0}^{\infty} V^{\otimes m}$ and $\mathbb{1}=\eta(1)$ is the unit element of $V$. Using the canonical filtration $T^{\leq n} V:=\bigoplus_{m=0}^{n} V^{\otimes m}$ of the tensor algebra, we define

$$
\phi_{!}(V)^{n}:=T^{\leq n} V /\left(T^{\leq n} V \cap \mathcal{I}\right)
$$

for all $n \geq 0$. This defines a sequential diagram

$$
\phi_{!}(V)^{0} \longleftrightarrow \phi_{!}(V)^{1} \longleftrightarrow \phi_{!}(V)^{2} \longleftrightarrow \cdots
$$

in the category $\mathbf{C h}(\mathbb{K})$, whose colimit

$$
\phi_{!}(V)=\operatorname{colim}_{n \geq 0}\left(\phi_{!}(V)^{n}\right)
$$

is the chain complex underlying $\phi_{!}(V)$. Using the explicit form of the relations (A.2), we observe that the quotient

$$
\phi_{!}(V)^{n+1} / \phi_{!}(V)^{n} \cong \widetilde{V}^{\otimes n+1} / \Sigma_{n+1}
$$

is given by the coinvariants of the canonical permutation group action on the $n+1$-fold tensor product of quotient chain complex $\widetilde{V}:=V / \mathbb{K} \mathbb{1}$. In other words, there is a short exact sequence

$$
0 \longrightarrow \phi_{!}(V)^{n} \longrightarrow \phi_{!}(V)^{n+1} \longrightarrow \widetilde{V}^{\otimes n+1} / \Sigma_{n+1} \longrightarrow 0
$$

of chain complexes, for all $n \geq 0$.

Let now $\rho: V \rightarrow V^{\prime}$ be a weak equivalence in $\mathbf{A l g}_{\text {uLie. }}$ Our goal is to prove that $\phi_{!}(\rho): \phi_{!}(V) \rightarrow \phi_{!}\left(V^{\prime}\right)$ is a weak equivalence in $\mathbf{A} \mathbf{I g}_{\text {As }}$, i.e. that the induced map $H_{\bullet}\left(\phi_{!}(\rho)\right): H_{\bullet}\left(\phi_{!}(V)\right) \rightarrow H_{\bullet}\left(\phi_{!}\left(V^{\prime}\right)\right)$ in homology is an isomorphism. Because filtered colimits commute with forming homologies, it is by (A.3) sufficient to prove that

$$
H_{\bullet}\left(\phi_{!}(\rho)^{n}\right): H_{\bullet}\left(\phi_{!}(V)^{n}\right) \longrightarrow H_{\bullet}\left(\phi_{!}\left(V^{\prime}\right)^{n}\right)
$$

is an isomorphism, for all $n \geq 0$. For $n=0$ this is clearly the case, and for $n=1$ it follows from the observation that the induced map $\rho: \widetilde{V}=V / \mathbb{K} \mathbb{1} \rightarrow \widetilde{V}^{\prime}=V^{\prime} / \mathbb{K} \mathbb{1}^{\prime}$ is a quasi-isomorphism. 
The case $n>1$ is proven by induction. Assume that (A.6) is an isomorphism for some $n \geq 0$. The short exact sequence (A.5) of chain complexes induces a long exact sequence in homology and $\rho: V \rightarrow V^{\prime}$ induces a map of long exact sequences

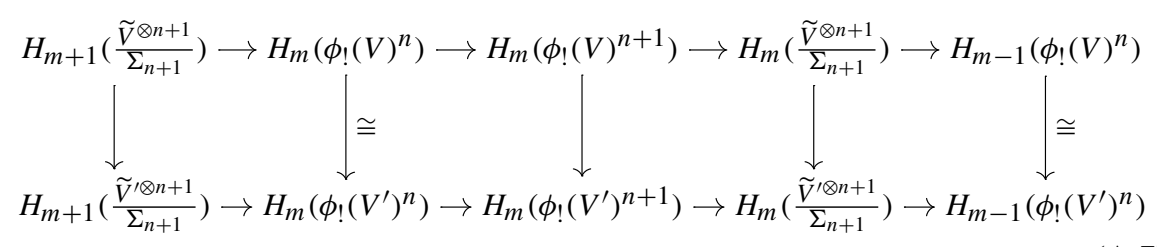

where by our induction hypothesis the second and fifth vertical maps are isomorphisms. The induction step consists of proving that the middle vertical map is an isomorphism. By the five lemma, this follows if the first and fourth vertical maps are isomorphisms. Because $\mathbb{K}$ is a field of characteristic zero, the chain complex $\widetilde{V}^{\otimes n+1} / \Sigma_{n+1}$ of coinvariants of the permutation group action is isomorphic to the chain complex $\left(\widetilde{V}^{\otimes n+1}\right)^{\Sigma_{n+1}}$ of invariants of the permutation group action. For char $\mathbb{K}=0$, taking invariants of finite group actions preserves quasi-isomorphisms, so the Künneth theorem and the fact that $\rho: \widetilde{V} \rightarrow \widetilde{V}^{\prime}$ is a quasi-isomorphism imply that the first and fourth vertical maps in (A.7) are isomorphisms. This completes the proof.

\section{References}

1. Bär, C., Ginoux, N., Pfäffle, F.: Wave Equations on Lorentzian Manifolds and Quantization. European Mathematical Society, Zürich, Switzerland (2007). arXiv:0806.1036 [math.DG]

2. Bär, C., Ginoux, N.: Classical and quantum fields on Lorentzian manifolds. Springer Proc. Math. 17, 359 (2011). arXiv:1104.1158 [math-ph]

3. Benini, M., Dappiaggi, C., Hack, T.P.: Quantum field theory on curved backgrounds: a primer. Int. J. Mod. Phys. A 28, 1330023 (2013). arXiv:1306.0527 [gr-qc]

4. Benini, M., Dappiaggi, C., Schenkel, A.: Algebraic quantum field theory on spacetimes with timelike boundary. Annales Henri Poincaré 19(8), 2401 (2018). arXiv:1712.06686 [math-ph]

5. Benini, M., Schenkel, A.: Poisson algebras for non-linear field theories in the Cahiers topos. Annales Henri Poincaré 18(4), 1435 (2017). arXiv:1602.00708 [math-ph]

6. Benini, M., Schenkel, A.: Higher Structures in Algebraic Quantum Field Theory. arXiv:1903.02878 [hep-th] (to appear in Fortschritte der Physik)

7. Benini, M., Schenkel, A., Schreiber, U.: The stack of Yang-Mills fields on Lorentzian manifolds. Commun. Math. Phys. 359(2), 765 (2018). arXiv:1704.01378 [math-ph]

8. Benini, M., Schenkel, A., Woike, L.: Operads for Algebraic Quantum Field Theory. arXiv:1709.08657 [math-ph]

9. Benini, M., Schenkel, A., Woike, L.: Homotopy theory of algebraic quantum field theories. Lett. Math. Phys. 109(7), 1487 (2019)

10. Berger, C., Moerdijk, I.: Resolution of coloured operads and rectification of homotopy algebras. In: Davydov, A., Batanin, M., Johnson, M., Lack, S., Neeman, A. (eds.) Categories in Algebra, Geometry and Mathematical Physics. Contemporary Mathematics, vol. 431, pp. 31-58. American Mathematical Society, Providence (2007)

11. Brunetti, R., Dappiaggi, C., Fredenhagen, K., Yngvason, J.: Advances in Algebraic Quantum Field Theory. Springer, Heidelberg (2015)

12. Brunetti, R., Fredenhagen, K., Ribeiro, P.L.: Algebraic Structure of Classical Field Theory I: Kinematics and Linearized Dynamics for Real Scalar Fields. arXiv:1209.2148 [math-ph]

13. Brunetti, R., Fredenhagen, K., Verch, R.: The generally covariant locality principle: a new paradigm for local quantum field theory. Commun. Math. Phys. 237, 31 (2003). arXiv:math-ph/0112041 
14. Collini, G.: Fedosov quantization and perturbative quantum field theory. Ph.D. thesis, Universität Leipzig (2016) arXiv:1603.09626 [math-ph]

15. Costello, K., Gwilliam, O.: Factorization Algebras in Quantum Field Theory: Volume 1, New Mathematical Monographs, vol. 31. Cambridge University Press, Cambridge (2017)

16. Dappiaggi, C., Murro, S., Schenkel, A.: Non-existence of natural states for Abelian Chern-Simons theory. J. Geom. Phys. 116, 119 (2017). arXiv:1612.04080 [math-ph]

17. Dwyer, W.G., Spalinski, J.: Homotopy theories and model categories. In: James, I.M. (ed.) Handbook of Algebraic Topology, vol. 73. North-Holland, Amsterdam (1995)

18. Fewster, C.J., Verch, R.: Algebraic quantum field theory in curved spacetimes. In: Brunetti, R., Dappiaggi, C., Fredenhagen, K., Yngvason, J. (eds.) Advances in algebraic quantum field theory, pp. 125-189. Springer, Heidelberg (2015). arXiv:1504.00586 [math-ph]

19. Fredenhagen, K.: Generalizations of the theory of superselection sectors. In: Kastler, D. (ed.) The Algebraic Theory of Superselection Sectors: Introduction and Recent Results, p. 379. World Scientific Publishing, Singapore (1990)

20. Fredenhagen, K.: Global observables in local quantum physics. In: Araki, H., Ito, K.R., Kishimoto, A., Ojima, I. (eds.) Quantum and Non-commutative Analysis: Past, Present and Future Perspectives, pp. 41-51. Kluwer Academic Publishers, Dordrecht (1993)

21. Fredenhagen, K., Rehren, K.-H., Schroer, B.: Superselection sectors with braid group statistics and exchange algebras II: geometric aspects and conformal covariance. Rev. Math. Phys. 4, 113 (1992)

22. Fredenhagen, K., Rejzner, K.: Batalin-Vilkovisky formalism in the functional approach to classical field theory. Commun. Math. Phys. 314, 93 (2012). arXiv:1101.5112 [math-ph]

23. Fredenhagen, K., Rejzner, K.: Batalin-Vilkovisky formalism in perturbative algebraic quantum field theory. Commun. Math. Phys. 317, 697 (2013). arXiv:1110.5232 [math-ph]

24. Gwilliam, O., Haugseng, R.: Linear Batalin-Vilkovisky quantization as a functor of $\infty$-categories. Selecta Mathematica 24, 1247 (2018). arXiv:1608.01290 [math.AT]

25. Haag, R., Kastler, D.: An algebraic approach to quantum field theory. J. Math. Phys. 5, 848 (1964)

26. Hawkins, E.: A cohomological perspective on algebraic quantum field theory. Commun. Math. Phys. 360(1), 439 (2018). arXiv:1612.05161 [math-ph]

27. Hinich, V.: Homological algebra of homotopy algebras. Commun. Algebra 25(10), 3291-3323 (1997) arXiv:q-alg/9702015. Erratum: arXiv:math/0309453 [math.QA]

28. Hinich, V.: Rectification of algebras and modules. Doc. Math. 20, 879-926 (2015). arXiv:1311.4130 [math.QA]

29. Hollands, S.: Renormalized quantum Yang-Mills fields in curved spacetime. Rev. Math. Phys. 20, 1033 (2008). arXiv:0705.3340 [gr-qc]

30. Hovey, M.: Model Categories, Mathematical Surveys and Monographs, vol. 63. The American Mathematical Society, Providence (1999)

31. Lang, B.: Universal constructions in algebraic and locally covariant quantum field theory. Ph.D. thesis, University of York (2014)

32. Ruzzi, G., Vasselli, E.: A new light on nets of $C^{*}$-algebras and their representations. Commun. Math. Phys. 312, 655 (2012). arXiv:1005.3178 [math.OA]

33. Schreiber, U.: Differential cohomology in a cohesive infinity-topos. https://ncatlab.org/schreiber/show/ differential+cohomology+in+a+cohesive+topos arXiv:1310.7930 [math-ph]

34. Yau, D.: Colored Operads, Graduate Studies in Mathematics, vol. 170. American Mathematical Society, Providence (2016)

Publisher's Note Springer Nature remains neutral with regard to jurisdictional claims in published maps and institutional affiliations. 